\title{
On the strong solutions of the Primitive Equations in 2D domains.
}

\author{
F. Guillén-González ${ }^{1}$ \& M.A. Rodríguez-Bellido² \\ Departamento de Ecuaciones Diferenciales y Análisis Numérico, Universidad de Sevilla. \\ c/ Tarfia s/n, 41012 Sevilla (SPAIN). \\ e-mails: guillen@numer.us.es, angeles@numer.us.es
}

Correspondence and proofs for corrections: F. Guillén-González

Key words: hydrostatic pressure, mixed boundary conditions, local and global existence, uniqueness, asymptotic behaviour.

\section{Introduction}

Some geophysical fluids can be modelled through the so-called "primitive equations" [1], [2]. This model is obtained formally from the Navier-Stokes equations, with anysotropic (eddy) viscosity, assuming two important simplifications: hydrostatic pressure (depending linearly on the depth) and the rigid lid hypothesis (fix water surface) [3] . For simplicity, we take constant density and assume that the effects due to the temperature (and salinity) can be decoupled from the dynamic of the flow. Then, we have a three-dimensional flow induced by the wind tension on the surface and by the centripetal and Coriolis forces. When the Earth curvature is not considered, we can use cartesian coordinates instead of spherical coordinates (see Lions-Teman-Wang [2] for the model with spherical coordinates), hence the domain is given by

$$
\Omega=\left\{(\vec{x}, z) \in \mathbb{R}^{3} ; \vec{x} \in \omega,-D(\vec{x})<z<0\right\},
$$

where $\omega \subseteq \mathbb{R}^{2}$ is an open domain and $D: \bar{\omega} \rightarrow \mathbb{R}_{+}$is the depth function. The different boundaries of $\Omega$ (surface, bottom and sidewalls) are respectively: $\Gamma_{s}=\{(\vec{x}, 0) ; \vec{x} \in \omega\}$, $\Gamma_{b}=\{(\vec{x},-D(\vec{x})) ; \vec{x} \in \omega\}$ and $\Gamma_{l}=\{(\vec{x}, z) ; \vec{x} \in \partial \omega,-D(\vec{x})<z<0\}$. Including, as it is usual ([4]), centripetal effects into the pressure term, the three-dimensional model is:

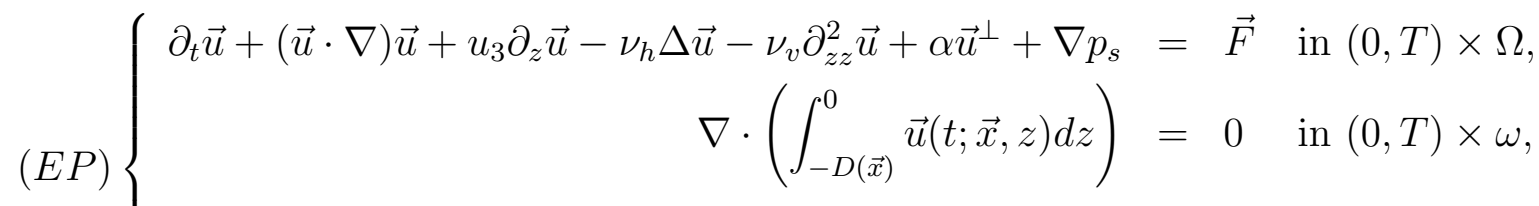

$$
\begin{aligned}
& \vec{u}_{\mid t=0}=\vec{u}_{0} \text { in } \Omega, \\
& \nu_{v} \partial_{z} \vec{u}_{\Gamma_{s}}=\vec{\tau}, \quad \vec{u}_{\mid \Gamma_{b} \cup \Gamma_{l}}=\overrightarrow{0} \quad \text { in }(0, T) .
\end{aligned}
$$

\footnotetext{
${ }^{1}$ Partially supported by C.I.C.Y.T project MAR98-0486

${ }^{2}$ Supported by C.I.C.Y.T project MAR98-0486
} 
Here, we denote $\vec{x}=(x, y), \nabla=\left(\partial_{x}, \partial_{y}\right)$ and $\Delta=\partial_{x x}^{2}+\partial_{y y}^{2}$. The unknowns are the horizontal component of flow velocity $\vec{u}=\left(u_{1}, u_{2}\right):(0, T) \times \Omega \rightarrow \mathbb{R}^{2}$ and the surface pressure $p_{s}:(0, T) \times \omega \rightarrow \mathbb{R}$, whereas the vertical component of the flow velocity is

$$
u_{3}(t ; \vec{x}, z)=-\int_{-D(\vec{x})}^{z} \nabla \cdot \vec{u}(t ; \vec{x}, s) d s, \quad \forall t \in(0, T), \quad \forall(\vec{x}, z) \in \Omega .
$$

Moreover, $\nu_{h}$ and $\nu_{v}>0$ are positive constants, representing horizontal and vertical (eddy) viscosity coefficients respectively, $\vec{F}:(0, T) \times \Omega \rightarrow \mathbb{R}^{2}$ is an horizontal external force field (depending on temperature and salinity, for instance) and $\vec{\tau}:(0, T) \times \Gamma_{s} \rightarrow \mathbb{R}^{2}$ represents the horizontal stress on the surface produced by the wind. Finally, $\alpha \vec{u}^{\perp}=\alpha\left(-u_{2}, u_{1}\right)$ models Coriolis effects, the no-slipt condition is assumed on the bottom and vertical slipting is permitted on the sidewalls.

To give a variational formulation to problem $(E P)$, let us define the following function spaces:

$$
\begin{gathered}
C_{b, l}^{\infty}(\Omega)=\left\{\vec{\varphi} \in C^{\infty}(\Omega)^{2} ; \operatorname{supp}(\vec{\varphi}) \text { is a compact set } \subseteq \bar{\Omega} \backslash\left(\Gamma_{b} \cup \Gamma_{l}\right)\right\}, \\
H_{b, l}^{1}(\Omega)=\bar{C}_{b, l}^{\infty} H^{1}(\Omega)=\left\{\vec{v} \in H^{1}(\Omega)^{2} ; \vec{v}=0 \text { on } \Gamma_{b} \cup \Gamma_{l}\right\}, \quad H_{b, l}^{-1}(\Omega)=\text { dual of } H_{b, l}^{1}(\Omega), \\
\mathcal{V}=\left\{\vec{\varphi} \in C_{b, l}^{\infty}(\Omega)^{2} ; \nabla \cdot\langle\vec{\varphi}\rangle=0 \text { in } \omega\right\}, \quad \text { where }\langle\vec{\varphi}\rangle(\vec{x})=\int_{-D(\vec{x})}^{0} \vec{\varphi}(\vec{x}, z) d z, \\
H=\overline{\mathcal{V}}^{L^{2}}=\left\{\vec{v} \in L^{2}(\Omega)^{2} ; \nabla \cdot\langle\vec{v}\rangle=0 \text { in } \omega,\langle\vec{v}\rangle \cdot \vec{n}_{\mid \partial \omega}=0\right\}, \\
V=\overline{\mathcal{V}}^{H^{1}}=\left\{\vec{v} \in H^{1}(\Omega)^{2} ; \nabla \cdot\langle\vec{v}\rangle=0 \text { in } \omega, \vec{v}_{\mid \Gamma_{b} \cup \Gamma_{l}}=\overrightarrow{0}\right\} .
\end{gathered}
$$

Definition 1.1 Let $\vec{u}_{0} \in H, \vec{F} \in L^{2}\left(0, T ; H_{b, l}^{-1}(\Omega)^{2}\right)$ and $\vec{\tau} \in L^{2}\left(0, T ; H^{-1 / 2}\left(\Gamma_{s}\right)^{2}\right)$. We say that $\vec{u}:(0, T) \times \Omega \rightarrow \mathbb{R}^{2}$ is a weak solution of $(E P)$ in $(0, T)$ if $\vec{u} \in L^{\infty}(0, T ; H) \cap$ $L^{2}(0, T ; V)$, verifies the variational formulation:

$$
\begin{aligned}
& \int_{0}^{T} \int_{\Omega}\left(-\vec{u} \cdot\left(\partial_{t} \vec{\varphi}+(\vec{u} \cdot \nabla) \vec{\varphi}+u_{3} \partial_{z} \vec{\varphi}\right)+\nu_{h} \nabla \vec{u}: \nabla \vec{\varphi}+\nu_{v} \partial_{z} \vec{u} \cdot \partial_{z} \vec{\varphi}+\alpha \vec{u}^{\perp} \cdot \vec{\varphi}\right) d \Omega d t \\
& =\int_{\Omega} \vec{u}_{0} \cdot \vec{\varphi}(0) d \Omega+\int_{0}^{T}\langle\vec{F}, \vec{\varphi}\rangle_{\Omega} d t+\int_{0}^{T}\langle\vec{\tau}, \vec{\varphi}\rangle_{\Gamma_{s}} d t, \quad \forall \vec{\varphi} \in C^{1}([0, T] ; \mathcal{V}) \text { s.t. } \vec{\varphi}(T)=\overrightarrow{0},
\end{aligned}
$$

and, moreover $\vec{u}$ satisfies the energy inequality:

$$
\begin{aligned}
& \frac{1}{2}\|\vec{u}\|_{L^{2}(\Omega)}^{2}+\int_{0}^{t}\left(\nu_{h}\|\nabla \vec{u}\|_{L^{2}(\Omega)}^{2}+\nu_{v}\left\|\partial_{z} \vec{u}\right\|_{L^{2}(\Omega)}^{2}\right) d s \\
& \quad \leq \frac{1}{2}\left\|\vec{u}_{0}\right\|_{L^{2}(\Omega)}^{2}+\int_{0}^{t}\langle\vec{F}, \vec{u}\rangle_{\Omega} d s+\int_{0}^{t}\langle\vec{\tau}, \vec{u}\rangle_{\Gamma_{s}} d s \quad \text { a.e. } t \in(0, T) .
\end{aligned}
$$

Here, $\langle\cdot, \cdot\rangle_{\Omega}$ denotes duality between $H_{b, l}^{-1}(\Omega)$ and $H_{b, l}^{1}(\Omega)$, whereas $\langle\cdot, \cdot\rangle_{\Gamma_{s}}$ denotes duality between $H^{-1 / 2}\left(\Gamma_{s}\right)$ and $H^{1 / 2}\left(\Gamma_{s}\right)$. 
Definition 1.2 Let $\vec{u}_{0} \in V, \vec{F} \in L^{2}\left(0, T ; L^{2}(\Omega)^{2}\right), \vec{\tau} \in L^{2}\left(0, T ; H^{1 / 2}\left(\Gamma_{s}\right)^{2}\right)$ and $\partial_{t} \vec{\tau} \in$ $L^{2}\left(0, T ; H^{-3 / 2}\left(\Gamma_{s}\right)^{2}\right)$. Let $\vec{u}$ be a weak solution of $(E P)$ in $(0, T)$, we say that $\vec{u}$ is a strong solution if verifies the additional regularity conditions:

$$
\vec{u} \in L^{\infty}(0, T ; V) \cap L^{2}\left(0, T ; H^{2}(\Omega)^{2} \cap V\right), \quad \partial_{t} \vec{u} \in L^{2}(0, T ; H) .
$$

The existence of a weak solution is well known, see Lewandovski [3] and Lions-TemanWang [2], always in domains with sidewalls (i.e. $D \geq D_{\min }>0$ in $\bar{\omega}$ ). In these works, compactness method is used to obtain the velocity $\vec{u}$ in a space with the restriction $\nabla \cdot\langle\vec{u}\rangle=0$ and the pressure is recovered, in the latter part of the argument, by a specific De Rham's lemma on the surface. In domains without sidewalls, the existence of a weak solution is obtained as a consequence of a limit process applied to the Navier-Stokes equations with anysotropic viscosity when the ratio depth over horizontal diameter (of the domain) tends to zero, see Besson-Laydi [5] for the stationary case and Azerad-Guillén [6] for the evolution case. Finally, the existence of a weak solution in domains without sidewalls can be proved by internal approximation arguments: a mixed (velocity-pressure) variational formulation of the stationary problem is approximated by a conform Finite Element method in Chacón-Guillén [7] and a semi-discretization in time of the evolution problem is proved that converges to continuous problem in Guillén-Redondo [8, 9].

However, to as far as we know, there are not results about the existence of strong solution of problem $(E P)$, excepting the stationary linear case [10]. One of the principal problems in this study is the treatment of the boundary conditions; on the surface we have a non homogeneous Neumann condition, whereas the sidewalls and the bottom have homogeneous Dirichlet condition. On the other hand, the uniqueness of the solution of problem $(E P)$ is also an open problem, even in the case of strong solutions.

\subsection{The $2 D$ problem}

In this work, we are going to consider mainly the two-dimensional problem (with only one horizontal direction). In this case, Coriolis forces have not sense. Now, the model is:

$$
\left(E P_{2}\right)\left\{\begin{aligned}
\partial_{t} u+u \partial_{x} u+u_{3} \partial_{z} u-\nu_{h} \partial_{x x}^{2} u-\nu_{v} \partial_{z z}^{2} u+\partial_{x} p_{s} & =F \text { in }(0, T) \times \Omega, \\
\partial_{x}\langle u\rangle & =0 \quad \text { in }(0, T) \times \omega, \\
u_{\left.\right|_{t=0}} & =u_{0} \text { in } \Omega, \\
\nu_{v} \partial_{z} u_{\left.\right|_{\Gamma_{s}}}=\tau, \quad u_{\Gamma_{\Gamma_{b}} \cup \Gamma_{l}} & =0 \quad \text { in }(0, T) .
\end{aligned}\right.
$$

Then, all the unknowns are scalar: the horizontal component of the flux velocity $u$ : $(0, T) \times \Omega \rightarrow \mathbb{R}$ and the superficial pressure $p_{s}:(0, T) \times \omega \rightarrow \mathbb{R}$. The vertical component of the flux velocity is $u_{3}(t ; x, z)=-\int_{-D(x)}^{z} \partial_{x} u(t ; x, s) d s$. One important difference respect to $3 \mathrm{D}$ case is that now $\omega \subseteq \mathbb{R}$ is an interval, which changes the function spaces of free divergence. Now, we have the following simpler characterizations:

$$
\mathcal{V}=\left\{\varphi \in C_{b, l}^{\infty}(\Omega) ;\langle\varphi\rangle=0 \text { in } \omega\right\},
$$




$$
\begin{gathered}
H=\left\{v \in L^{2}(\Omega) ;\langle v\rangle=0 \text { in } \omega\right\}, \\
V=\left\{v \in H^{1}(\Omega) ;\langle v\rangle=0 \text { in } \omega, v_{\left.\right|_{\Gamma_{b}} \cup \Gamma_{l}}=0\right\} .
\end{gathered}
$$

Finally, definitions of weak and strong solutions are similar to the 3D case (changing vectorial notation by scalar notation in $u$ and $x$, and vanishing the Coriolis term).

Remark 1.1 Now, the 2nd. equation in $\left(E P_{2}\right)$ means that $\langle u\rangle$ only depends on $t$.

\subsection{Main results}

In this paper, we will obtain the following main results, all in the $2 \mathrm{D}$ case and in domains with sidewalls.

Theorem 1.3 (Strong global solution for small data.) Let $\omega \subseteq \mathbb{R}$ an interval and $D \in C^{2}(\bar{\omega})$ such that $D \geq D_{\text {min }}>0$ in $\bar{\omega}$. We assume $u_{0} \in V, F \in L^{2}\left(0, T ; L^{2}(\Omega)\right)$ and $\tau \in L^{2}\left(0, T ; H_{0}^{1 / 2+\varepsilon}\left(\Gamma_{s}\right)\right)$, for some $\varepsilon>0$, with $\partial_{t} \tau \in L^{2}\left(0, T ; H^{-1 / 2}\left(\Gamma_{s}\right)\right)$. If the following "smallness restriction" is assumed: $\forall t \in[0, T]$,

$$
(H)\left\{\begin{aligned}
\exp & \left(-\frac{1}{4 C} t+\int_{0}^{t} a(s) d s\right)\left\{2\left(\left\|u_{0}\right\|_{V}^{2}+C^{2}\|\tau(0)\|_{H^{-1 / 2}\left(\Gamma_{s}\right)}^{2}\right)\right. \\
& \left.+\int_{0}^{t} \exp \left(\frac{1}{4 C} s-\int_{0}^{s} a(\sigma) d \sigma\right) b(s) d s\right\}<M^{2}
\end{aligned}\right.
$$

where $M$ is a small enough positive constant (see Lemma 5.2), $C$ is a constant that appears in (11) and $a, b$ are functions depending on the data $\tau$ and $F$ (see (30) and (31)), then there exists a unique strong solution $\left(u, p_{s}\right)$ of $\left(E P_{2}\right)$ in $(0, T)\left(p_{s}\right.$ is unique up to a function of $t$ ).

Corollary 1.4 (Asymptotic behaviour when $t \uparrow+\infty$.) Let $\omega \subseteq \mathbb{R}$ an interval and $D \in C^{2}(\bar{\omega})$ such that $D \geq D_{\min }>0$ in $\bar{\omega}$. We assume $u_{0} \in V, F \in L^{2}\left(0,+\infty ; L^{2}(\Omega)\right)$ and $\tau \in L^{2}\left(0,+\infty ; H_{0}^{1 / 2+\varepsilon}\left(\Gamma_{s}\right)\right)$, for some $\varepsilon>0$, with $\partial_{t} \tau \in L^{2}\left(0,+\infty ; H^{-1 / 2}\left(\Gamma_{s}\right)\right)$. If the "smallness restriction" $(H)$ is assumed $\forall t \in[0,+\infty)$, then there exist a unique strong solution $\left.u \in L^{2}\left(0,+\infty ; H^{2}(\Omega) \cap V\right)\right) \cap L^{\infty}(0,+\infty ; V), \partial_{t} u \in L^{2}(0,+\infty ; H)$. Moreover, if

$$
\int_{0}^{+\infty} \exp \left(\frac{1}{4 C} t\right)\left\{\|\tau(t)\|_{H_{0}^{1 / 2+\varepsilon}\left(\Gamma_{s}\right)}^{2}+\left\|\partial_{t} \tau(t)\right\|_{H^{-1 / 2}\left(\Gamma_{s}\right)}^{2}+\|F(t)\|_{L^{2}(\Omega)}^{2}\right\} d t<+\infty
$$

there exists two constants $K_{1}, K_{2}>0$ such that:

$$
\|\nabla u(t)\|_{L^{2}(\Omega)}^{2} \leq \exp \left(-\frac{1}{4 C} t\right) K_{1}\left(2\left\|u_{0}\right\|_{V}^{2}+C^{2}\|\tau(0)\|_{H^{-1 / 2}\left(\Gamma_{s}\right)}^{2}+K_{2}\right) \quad \forall t \geq 0,
$$

(i.e. the solution vanishes exponentially in the $H^{1}(\Omega)$-norm, as $t$ increases). 
Theorem 1.5 (Strong local solution for any data.) Under hypotheses of Theorem 1.3 , changing the restriction $(H)$ by $D_{\text {max }}\left(=\max _{\bar{\omega}} D\right)$ small enough, then there exists $T_{*} \in(0, T]$ and a unique strong solution $\left(u, p_{s}\right)$ of $\left(E P_{2}\right)$ in $\left(0, T_{*}\right)$.

Theorem 1.6 (Uniqueness of strong/weak solution.) Let $u$ be a weak solution of $\left(E P_{2}\right)$ in $(0, T)$. If there exists another weak solution $\bar{u}$ of $\left(E P_{2}\right)$ in $(0, T)$, such that verifies the additional regularity:

$$
\partial_{z} \bar{u} \in L^{4}\left(0, T ; L^{4}(\Omega)\right),
$$

then both solutions coincide in $(0, T)$.

Remark 1.2 The arguments to prove all these main results, will not be valid in the $3 D$ case. On the other hand, the additional regularity (6) that implies uniqueness is verified by the strong solutions of $\left(E P_{2}\right)$ (and not by only weak solutions). Applying this uniqueness argument to the $3 D$ case, it is necessary an additional regularity that is not verified by the strong solutions.

To make the study about existence of strong solutions, it will be convenient to decompose the problem $(E P)$ in two: one linear problem $(L)$ with nonhomogeneous boundary conditions on the surface, and a nonlinear problem $(P)$ with homogeneous boundary conditions.

This paper is organized as follows. In Section 2, we prove some technical inequalities that we will use in the following.

The linear problem $(L)$ is studied in Sections 3 and 4, whereas the study of $(P)$ (by means of a Galerkin method) is made in Section 5, where the proof of Theorem 1.3 is finished. Indeed, in Section 3, using the known results ([10]) about strong solution of the linear stationary problem $\left(L_{s t}\right)$, we deduce some properties of the differential operator associated, that we apply in Section 4, arriving at the existence and uniqueness of strong solution of $(L)$ (all these results are valid in any space dimension).

In Section 6, we present the proof of Theorem 1.5, based in a fixed point argument (in particular, it is not possible to make a Galerkin argument as in Theorem 1.3). Finally, the uniqueness of weak solution assuming that a strong solution exists (Theorem 1.6), is proved in Section 7.

\section{Some technical results}

First, we see three technical lemmas that we will used several times in this paper:

Lemma 2.1 Let $\Omega \subseteq \mathbb{R}^{N}$ ( $N=2$ or 3 ) be the domain considered in this work (defined by (1)). Then, for all $\vec{v} \in W^{1, p}(\Omega)^{N-1}(p>1)$, if we define $v_{3}(\vec{x}, z)=-\int_{-D(\vec{x})}^{z} \nabla \cdot \vec{v}(\vec{x}, s) d s$, one has:

$$
\left\|v_{3}\right\|_{L^{p}(\Omega)} \leq D_{\max }\|\nabla \cdot \vec{v}\|_{L^{p}(\Omega)}
$$


Proof: It is a consequence of Fubini's Theorem:

$$
\begin{aligned}
\left\|v_{3}\right\|_{L^{p}(\Omega)}^{p} & =\int_{\Omega}\left|\int_{-D(\vec{x})}^{z} \nabla \cdot \vec{v}(\vec{x}, s) d s\right|^{p} d \Omega d z \\
& \leq \int_{\Omega}\left(\int_{-D(\vec{x})}^{z}|\nabla \cdot \vec{v}(\vec{x}, s)|^{p} d s\right)(z+D(\vec{x}))^{p / p^{\prime}} d \Omega d z \\
& =\int_{\omega} \int_{-D(\vec{x})}^{0}|\nabla \cdot \vec{v}(\vec{x}, s)|^{p}\left(\int_{s}^{0}(z+D(\vec{x}))^{p / p^{\prime}} d z\right) d \Omega d s \\
& \leq \frac{D_{\max }^{p}}{p} \int_{\omega} \int_{-D(\vec{x})}^{0}|\nabla \cdot \vec{v}(\vec{x}, z)|^{p} d \Omega d s=\frac{D_{\max }^{p}}{p}\|\nabla \cdot \vec{v}\|_{L^{p}(\Omega)}^{p}
\end{aligned}
$$

Lemma 2.2 (Interpolation inequalities.) Let $\Omega \subseteq \mathbb{R}^{N}$ be a Lipschitz-continuous domain. The following inequality holds:

$$
\|\vec{u}\|_{L^{p}(\Omega)} \leq C\|\vec{u}\|_{W^{1, N}(\Omega)}^{1-q / p}\|\vec{u}\|_{L^{q}(\Omega)}^{q / p}, \quad \forall \vec{u} \in W^{1, N}(\Omega)^{N-1},
$$

where $N \leq q \leq p<+\infty$.

Proof: It is taken from the Nirenberg's paper [11], where is proved the result when $\Omega=\mathbb{R}^{N}$. Here, we adapt the proof to a Lipschitz-continuous domain $\Omega$.

For this, we pass these inequalities to $\Omega$ using a prolongation operator [12]

$$
E: W^{1,1}(\Omega)^{N-1} \longrightarrow W^{1, N}\left(\mathbb{R}^{N}\right)^{N-1},
$$

verifying $E \vec{u}_{\left.\right|_{\Omega}}=\vec{u}$ and $\|E \vec{u}\|_{W^{1, N}\left(\mathbf{R}^{N}\right)} \leq C\|\vec{u}\|_{W^{1, N}(\Omega)}, \forall \vec{u} \in W^{1, N}(\Omega)^{N-1}$, for some $C=C(\Omega)>0$. Nirenberg's result says

$$
\|E \vec{u}\|_{L^{p}\left(\mathbf{R}^{N}\right)} \leq C\|E \vec{u}\|_{W^{1, N}\left(\mathbf{R}^{N}\right)}^{1-q / p}\|E \vec{u}\|_{L^{q}\left(\mathbf{R}^{N}\right)} .
$$

Therefore, since $\|\vec{u}\|_{L^{p}(\Omega)} \leq\|E \vec{u}\|_{L^{p}\left(\mathbf{R}^{N}\right)}$ and $\|E \vec{u}\|_{L^{q}\left(\mathbf{R}^{N}\right)} \leq C\|\vec{u}\|_{L^{q}(\Omega)}$, we arrive at (7).

An easy application to the above Lemma and the Poincaré's inequality, give us the following:

Corollary 2.3 Let $\Omega \subseteq \mathbb{R}^{N}$ be the domain considered in this work. The following inequality holds:

$$
\|\vec{u}\|_{L^{p}(\Omega)} \leq C\|\nabla \vec{u}\|_{L^{N}(\Omega)}^{1-q / p}\|\vec{u}\|_{L^{q}(\Omega)}^{q / p} \quad \forall \vec{u} \in W_{b, l}^{1, N}(\Omega)^{N-1},
$$

where $N \leq q \leq p<+\infty$.

Remark 2.1 The main advantage of the 2D case is to consider (7) and (8) for $N=2$. In the following, we will call Gagliardo-Nirenberg's inequality to (7) or (8) in the case $N=2, p=4$ and $q=2$, i.e.

$$
\begin{aligned}
\|u\|_{L^{4}(\Omega)} & \leq C\|u\|_{L^{2}(\Omega)}^{1 / 2}\|u\|_{H^{1}(\Omega)}^{1 / 2} \quad \forall u \in H^{1}(\Omega), \\
\|u\|_{L^{4}(\Omega)} & \leq C\|u\|_{L^{2}(\Omega)}^{1 / 2}\|\nabla u\|_{L^{2}(\Omega)}^{1 / 2} \quad \forall u \in H_{b, l}^{1}(\Omega) .
\end{aligned}
$$




\section{The stationary linear case}

In this Section, we will see some preliminary results about the linear stationary system (also called hydrostatic Stokes system):

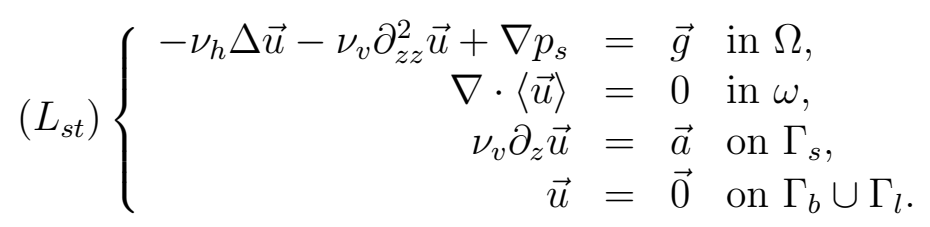

\subsection{Known results about existence and uniqueness}

Lemma 3.1 (Weak solution of $\left(L_{s t}\right)$ ) Let $\omega \subseteq \mathbb{R}^{d}\left(d=1\right.$ or 2 ) and let $\Omega \subseteq \mathbb{R}^{d+1}$, defined as in (1), be a Lipschitz-continuous domain. If $\vec{g} \in H_{b, l}^{-1}(\Omega)^{d}$ and $\vec{a} \in H^{-\overline{1} / 2}\left(\Gamma_{s}\right)^{d}$, then the problem $\left(L_{s t}\right)$ has a unique solution $\vec{u} \in H^{1}(\Omega)^{d}$. Moreover, one has the continuous dependence, i.e. there exists a constant $C=C\left(\Omega, \nu_{h}, \nu_{v}\right)>0$ such that

$$
\|\vec{u}\|_{V} \leq C\left\{\|\vec{a}\|_{H^{-1 / 2}\left(\Gamma_{s}\right)}+\|\vec{g}\|_{H_{b, l}^{-1}(\Omega)}\right\} .
$$

In [5], [7] and [3], there are different proofs of this result (even in the nonlinear case).

Lemma 3.2 (Strong solution of $\left(L_{s t}\right)$ ) Let $\omega \subseteq \mathbb{R}^{d}(d=1$ or 2$)$ be a $C^{2}$ domain and $D \in C^{2}(\bar{\omega})$ with $D \geq D_{\text {min }}>0$ in $\bar{\omega}$. If $\vec{g} \in L^{2}(\Omega)^{d}$ and $\vec{a} \in H_{0}^{1 / 2+\varepsilon}\left(\Gamma_{s}\right)^{d}$, for some $\varepsilon>0$, then the unique solution $\vec{u}$ of the problem $\left(L_{\text {st }}\right)$ belongs to $H^{2}(\Omega)^{d} \cap V$. Moreover, we have the continuous dependence, i.e. there exists a constant $C=C\left(\Omega, \nu_{h}, \nu_{v}\right)>0$ such that:

$$
\|\vec{u}\|_{H^{2}(\Omega)} \leq C\left\{\|\vec{a}\|_{H_{0}^{1 / 2+\varepsilon}\left(\Gamma_{s}\right)}+\|\vec{g}\|_{L^{2}(\Omega)}\right\} .
$$

See [10] for the proof of regularity. The continuous dependence can be deduced following the construction of the auxiliary problems made by Ziane in [10].

\subsection{The hydrostatic Stokes operator}

We define $A$, that it will call "hydrostatic Stokes operator", as the resolvent operator related to the homogeneous Neumann boundary conditions on the surface and Dirichlet boundary conditions on the bottom and sidewalls, i.e. $A: V \rightarrow V^{\prime}$ such that

$$
\langle A \vec{u}, \vec{v}\rangle_{V^{\prime}, V}=\int_{\Omega}\left(\nu_{h} \nabla \vec{u}: \nabla \vec{v}+\nu_{v} \partial_{z} \vec{u} \cdot \partial_{z} \vec{v}\right) d \Omega \quad \forall \vec{u}, \vec{v} \in V .
$$

Then, if we denote $\vec{g}=A \vec{u} \in V^{\prime}$, from Lemma $3.1, \vec{u}$ is the unique weak solution of the hydrostatic Stokes problem $\left(L_{s t}\right)$, with $\vec{a}=\overrightarrow{0}$. Moreover, taking into account Lemma 3.2, $A$ is a self-adjoint isomorphism from $H^{2}(\Omega)^{2} \cap V$ to $H$. In particular, if $A \vec{u}=\vec{g}$ with $\vec{g} \in H, \vec{u}$ is characterized as the unique strong solution of the problem $\left(L_{s t}\right)$, with $\vec{a}=\overrightarrow{0}$. Finally, the domain of $A$, defined by

$$
D(A)=\{\vec{u} ; \vec{u} \in V \text { and } A \vec{u} \in H\} .
$$

can be characterized as follows: 
Lemma 3.3 Let $\omega \subseteq \mathbb{R}^{d}(d=1$ or 2$)$ be a $C^{2}$ domain and $D \in C^{2}(\bar{\omega})$ with $D \geq D_{\text {min }}>0$ in $\bar{\omega}$. Then

$$
D(A)=\left\{\vec{u} ; \vec{u} \in H^{2}(\Omega)^{d} \cap V \text { and } \partial_{z} \vec{u}=\overrightarrow{0} \text { on } \Gamma_{s}\right\} .
$$

Moreover, there exists $C=C\left(\Omega, \nu_{h}, \nu_{v}\right)>0$ sucht that

$$
\|\vec{u}\|_{H^{2}(\Omega)} \leq C\|A \vec{u}\|_{L^{2}(\Omega)} \quad \forall \vec{u} \in D(A) .
$$

Proof: Let $Y$ be the right hand side of (14).

a) $D(A) \subset Y$ : Let $\vec{u} \in D(A)$. If we denote $\vec{g}=A \vec{u}$, then $\vec{u}$ is the weak solution of $\left(L_{s t}\right)$ with $\vec{a}=\overrightarrow{0}$. As $\vec{g} \in H$, from the Ziane's regularity results [10], we deduce that $\vec{u} \in Y$, and the continuous dependence (12) says:

$$
\|\vec{u}\|_{H^{2}(\Omega)} \leq C\|\vec{g}\|_{L^{2}(\Omega)}=C\|A \vec{u}\|_{L^{2}(\Omega)}
$$

b) $Y \subset D(A)$ : Let $\vec{u} \in Y$. If we denote $\vec{f}=-\nu_{h} \Delta \vec{u}-\nu_{v} \partial_{z z}^{2} \vec{u}$, then $\vec{f} \in L^{2}(\Omega)^{d}$ and $A \vec{u}=P \vec{f}$, where $P$ is the ortogonal projection from $L^{2}(\Omega)^{d}$ onto $H$. Hence $A \vec{u} \in H$, i.e. $\vec{u} \in D(A)$.

\subsection{Construction of a special basis}

In this subsection, we will prove the following result:

Lemma 3.4 Under the conditions of Lemma 3.3, there exists a sequence $\left\{\lambda_{j}\right\}_{j \geq 1} \subseteq \mathbb{R}$ with $0<\lambda_{1} \leq \lambda_{2} \leq \ldots \leq \lambda_{j} \leq \lambda_{j+1} \leq \ldots,\left\{\lambda_{j}\right\} \rightarrow+\infty$, and an orthonormal basis of $H$, $\left\{\vec{w}^{j}\right\}_{j \geq 1}$, where each $\vec{w}^{j}$ is an eigenfunction of $A$ associated to eigenvalue $\lambda_{j}$.

Proof: Let $\Lambda: H \longrightarrow D(A) \hookrightarrow H$ be the operator that associates each $\vec{g} \in H$ to $\vec{u} \in D(A)$, the unique strong solution of the problem $\left(L_{s t}\right)$ with $\vec{a}=\overrightarrow{0}$ (i.e. $A \vec{u}=\vec{g}$ ). This is an compact (using Lemma 3.3 and the compact embedding of $H^{2}(\Omega)^{2} \cap V$ into $H$ ) and self-adjoint operator

$$
\left(\Lambda \vec{g}_{1}, \vec{g}_{2}\right)=\left(\vec{u}_{1}, \vec{g}_{2}\right)=\left(\vec{u}_{1}, A \vec{u}_{2}\right)=\left(A \vec{u}_{1}, \vec{u}_{2}\right)=\left(\vec{g}_{1}, \Lambda \vec{g}_{2}\right) .
$$

Then, as $H$ is separable, we can apply the Hilbert Schmidt's Theorem (of spectral decomposition), and there exists an orthogonal basis of $H$ formed by eigenfunctions of $\Lambda$, $\left\{\vec{v}^{j}\right\}_{j \geq 1}\left(\Lambda \vec{v}^{j}=\mu_{j} \vec{v}^{j}\right.$, where $\mu_{j} \searrow 0$ as $\left.j \nearrow+\infty\right)$. Let $\lambda_{j}=1 / \mu_{j}$ and $\vec{z}^{j}=\mu_{j} \vec{v}^{j}$. Then $A \vec{z}^{j}=\lambda_{j} \vec{z}^{j}$, and the sequence $\vec{w}^{j}=\vec{z}^{j} / \sqrt{\lambda_{j}}$ is the orthonormal basis of the Lemma.

\section{The evolution linear case}

In this section we will study the strong solution of the nonstationary linear problem:

$$
(L)\left\{\begin{aligned}
\partial_{t} \vec{v}-\nu_{h} \Delta \vec{v}-\nu_{v} \partial_{z z}^{2} \vec{v}+\nabla q_{s} & =\vec{f} & & \text { in }(0, T) \times \Omega, \\
\nabla \cdot\langle\vec{v}\rangle & =0 & & \text { in }(0, T) \times \omega, \\
\vec{v}_{\mid t=0} & =\vec{v}_{0} & & \text { in } \Omega, \\
\nu_{v} \partial_{z} \vec{v} & =\vec{\tau} & & \text { on }(0, T) \times \Gamma_{s}, \\
\vec{v} & =\overrightarrow{0} & & \text { on }(0, T) \times\left(\Gamma_{b} \cup \Gamma_{l}\right) .
\end{aligned}\right.
$$


Theorem 4.1 Let $\omega \subseteq \mathbb{R}^{d}(d=1$ or 2$)$ be a $C^{2}$ domain and $D \in C^{2}(\bar{\omega})$ with $D \geq$ $D_{\text {min }}>0$ in $\bar{\omega}$. If $\vec{f} \in L^{2}((0, T) \times \Omega)^{d}, \vec{v}_{0} \in V, \vec{\tau} \in L^{2}\left(0, T ; H_{0}^{1 / 2+\varepsilon}\left(\Gamma_{s}\right)^{d}\right)$, for some $\varepsilon>0$, with $\partial_{t} \vec{\tau} \in L^{2}\left(0, T ; H^{-1 / 2}\left(\Gamma_{s}\right)^{d}\right)$, then there exists a unique strong solution $\vec{v}$ of $(L)$ in $(0, T)$. Moreover, there exists $C>0$ sucht that

$$
\begin{gathered}
\|\vec{v}\|_{L^{\infty}(V)}^{2}+\|\vec{v}\|_{L^{2}(D(A))}^{2}+\left\|\partial_{t} \vec{v}\right\|_{L^{2}(H)}^{2} \leq C\left\{\left\|\vec{v}_{0}\right\|_{V}^{2}+\|\vec{\tau}(0)\|_{H^{-1 / 2}\left(\Gamma_{s}\right)}^{2}\right. \\
\left.+\|\vec{f}\|_{L^{2}\left(L^{2}(\Omega)\right)}^{2}+\|\vec{\tau}\|_{L^{2}\left(H_{0}^{1 / 2+\varepsilon}\left(\Gamma_{s}\right)\right)}^{2}+\left\|\partial_{t} \vec{\tau}\right\|_{L^{2}\left(H^{-1 / 2}\left(\Gamma_{s}\right)\right)}^{2}\right\}
\end{gathered}
$$

Proof: Uniqueness can be easily deduced from the linearity of the problem $(L)$. The proof of the existence will be separate in several steps.

Step 1. Weak solution of $(L)$. The weak solution $\vec{v}$ of $(L)$ in $(0, T)$ can be obtained as a limit of Galerkin approximations $\vec{v}_{m} \in C^{1}\left([0, T] ; V_{m}\right)$ (being $V_{m}$ a $m$-dimensional subspace of $V$ ) such that

$$
(L)_{m}\left\{\begin{array}{c}
\frac{d}{d t} \int_{\Omega} \vec{v}_{m} \cdot \vec{\varphi} d \Omega+\nu_{h} \int_{\Omega} \nabla \vec{v}_{m}: \nabla \vec{\varphi} d \Omega+\nu_{v} \int_{\Omega} \partial_{z} \vec{v}_{m} \cdot \partial_{z} \vec{\varphi} d \Omega \\
=\int_{\Omega} \vec{f}_{m} \cdot \vec{\varphi} d \Omega+\left.\int_{\Gamma_{s}} \vec{\tau}_{m} \cdot \vec{\varphi}\right|_{\Gamma_{s}} d \sigma \quad \forall \vec{\varphi} \in V_{m} \\
\vec{v}_{m}(0) \quad \text { being the projection of } \vec{v}_{0} \text { onto } V_{m}
\end{array}\right.
$$

where $\vec{f}_{m} \in C^{0}\left([0, T] ; H_{b, l}^{-1}(\Omega)^{2}\right)$ and $\vec{\tau}_{m} \in C^{0}\left([0, T] ; H^{-1 / 2}\left(\Gamma_{s}\right)^{2}\right)$ are respectively regular approximations to $\vec{f}$ and $\vec{\tau}$.

Taking $\vec{v}_{m}$ as test function in $(L)_{m}$, one can deduce that the sequence $\vec{v}_{m}$ is bounded in $L^{\infty}(0, T ; H) \cap L^{2}(0, T ; V)$. Passing to the limit in a standard way, we obtain the weak regularity for $\vec{v}$.

Remark 4.1 (Weak solution of $(E P)$ ). Galerkin approximations of nonliner problem $(E P)$ are similar to problem $(L)_{m}$. The only differences are the nonlinear terms:

$$
\int_{\Omega}\left\{\left(\vec{u}_{m} \cdot \nabla\right) \vec{u}_{m}+u_{m 3} \partial_{z} \vec{u}_{m}\right\} \cdot \vec{\varphi} d \Omega
$$

where $u_{m 3}$ is defined from $\nabla \cdot \vec{u}_{m}$ as in (2). But, these terms vanish when $\vec{u}_{m}$ is taken as test function, hence we can also deduce that $\vec{u}_{m}$ is bounded in $L^{\infty}(0, T ; H) \cap L^{2}(0, T ; V)$. Now, by using a compactness result (estimating $\partial_{t} \vec{u}_{m}$ in a convenient space), we could pass to the limit and obtain a weak solution $\vec{u}$ of $\left(E P_{2}\right)$ in $(0, T)$.

Step 2. "Lifting" of the Neumann boundary conditions. We define the operator $B: \vec{a} \in H^{-1 / 2}\left(\Gamma_{s}\right)^{d} \rightarrow \vec{u}=B \vec{a} \in V$, where $\vec{u}$ is the weak solution of the hydrostatic Stokes problem $\left(L_{s t}\right)$ with $\vec{g}=\overrightarrow{0}$, i.e.

$$
\vec{u} \in V \quad \text { such that } \quad\langle A \vec{u}, \vec{\psi}\rangle_{V^{\prime}, V}=\langle\vec{a}, \vec{\psi}\rangle_{\Gamma_{s}} \quad \forall \vec{\psi} \in V
$$


Then, let us define

$$
\vec{e}(t)=B(\vec{\tau}(t)) \quad \text { a.e. } t \in(0, T) .
$$

From Lemma 3.2, since $\vec{\tau}(t) \in H_{0}^{1 / 2+\varepsilon}\left(\Gamma_{s}\right)^{d}$ a.e. $t \in(0, T)$, we have that $\vec{e}(t) \in H^{2}(\Omega)^{d} \cap V$ a.e. $t \in(0, T)$, and

$$
\|\vec{e}(t)\|_{H^{2}(\Omega)} \leq C\|\vec{\tau}(t)\|_{H_{0}^{1 / 2+\varepsilon}\left(\Gamma_{s}\right)} .
$$

Therefore, $\vec{e} \in L^{2}\left(0, T ; H^{2}(\Omega)^{d} \cap V\right)$ and

$$
\|\vec{e}\|_{L^{2}\left(H^{2}(\Omega)\right)} \leq C\|\vec{\tau}\|_{L^{2}\left(H_{0}^{1 / 2+\varepsilon}\left(\Gamma_{s}\right)\right)} .
$$

On the other hand, we have

$$
\partial_{t} \vec{e}=B\left(\partial_{t} \vec{\tau}(t)\right) \quad \text { a.e. } t \in(0, T) .
$$

To prove this one, we define $\vec{g}(t)=B\left(\partial_{t} \vec{\tau}(t)\right)$ and

$$
\vec{v}_{\delta}(t)=\frac{\vec{e}(t+\delta)-\vec{e}(t)}{\delta}-\vec{g}(t)=B\left(\frac{\vec{\tau}(t+\delta)-\vec{\tau}(t)}{\delta}-\partial_{t} \vec{\tau}(t)\right) .
$$

From Lemma 3.1, for $\vec{a}=\frac{\vec{\tau}(t+\delta)-\vec{\tau}(t)}{\delta}-\partial_{t} \vec{\tau}(t)$,

$$
\left\|\vec{v}_{\delta}(t)\right\|_{V} \leq C\left\|\frac{\vec{\tau}(t+\delta)-\vec{\tau}(t)}{\delta}-\partial_{t} \vec{\tau}(t)\right\|_{H^{-1 / 2}\left(\Gamma_{s}\right)} .
$$

The last expression tends to zero when $\delta \rightarrow 0$, hence, $\left\|\vec{v}_{\delta}(t)\right\|_{H^{1}(\Omega)} \rightarrow 0$. Therefore, we conclude (18). Moreover, Lemma 3.1 for $\vec{a}=\partial_{t} \vec{\tau}(t)$ says

$$
\left\|\partial_{t} \vec{e}(t)\right\|_{V} \leq C\left\|\partial_{t} \vec{\tau}(t)\right\|_{H^{-1 / 2}\left(\Gamma_{s}\right)} \quad \text { a.e. } t \in(0, T) .
$$

Therefore, as $\partial_{t} \vec{\tau} \in L^{2}\left(0, T ; H^{-1 / 2}\left(\Gamma_{s}\right)\right)$, then $\partial_{t} \vec{e} \in L^{2}(0, T ; V)$ and

$$
\left\|\partial_{t} \vec{e}\right\|_{L^{2}(V)} \leq C\left\|\partial_{t} \vec{\tau}\right\|_{L^{2}\left(H^{-1 / 2}\left(\Gamma_{s}\right)\right)} .
$$

In particular, $\vec{e} \in C^{0}([0, T] ; V)$. Moreover, from (19),

$$
\begin{aligned}
\|\vec{e}\|_{L^{\infty}(V)}^{2} & \leq C\left\{\|\vec{e}(0)\|_{V}^{2}+\|\vec{e}\|_{L^{2}(V)}\left\|\partial_{t} \vec{e}\right\|_{L^{2}(V)}\right\} \\
& \leq C\left\{\|\vec{\tau}(0)\|_{H^{-1 / 2}\left(\Gamma_{s}\right)}^{2}+\|\vec{\tau}\|_{L^{2}\left(H^{-1 / 2}\left(\Gamma_{s}\right)\right)}^{2}+\left\|\partial_{t} \vec{\tau}\right\|_{L^{2}\left(H^{-1 / 2}\left(\Gamma_{s}\right)\right)}^{2}\right\} .
\end{aligned}
$$

Step 3. Strong solution of the resulting problem (with homogeneous boundary conditions). If we decompose the solution $\vec{v}$ of $(L)$ as $\vec{v}=\vec{e}+\vec{y}$, where $\vec{e}$ is the regular function furnished in Step 2, then $\vec{y}$ is the solution of the resulting problem:

$$
(R)\left\{\begin{array}{rll}
\partial_{t} \vec{y}-\nu_{h} \Delta \vec{y}-\nu_{v} \partial_{z z}^{2} \vec{y}+\nabla \pi_{s} & =\vec{h} & \text { in }(0, T) \times \Omega, \\
\nabla \cdot\langle\vec{y}\rangle & =0 \quad \text { in }(0, T) \times \omega, \\
\left.\vec{y}\right|_{t=0} & =\vec{y}_{0} \quad \text { in } \Omega, \\
\nu_{v} \partial_{z} \vec{y} & =\overrightarrow{0} \quad \text { on }(0, T) \times \Gamma_{s}, \\
\vec{y} & =\overrightarrow{0} \quad \text { on }(0, T) \times\left(\Gamma_{b} \cup \Gamma_{l}\right),
\end{array}\right.
$$


where $\vec{h}=\vec{f}-\partial_{t} \vec{e}+\nu_{h} \Delta \vec{e}+\nu_{v} \partial_{z z}^{2} \vec{e} \in L^{2}\left(0, T ; L^{2}(\Omega)^{d}\right)$ and $\vec{y}_{0}=\vec{v}_{0}-\vec{e}(0) \in V$. Obviously, the proof of Theorem 4.1 will be finish if we show existence of strong solution $\vec{y}$ of the problem $(R)$ in $(0, T)$.

Let $V_{m}$ the subspace $V$ generated by $\left\{w^{1}, \ldots, w^{m}\right\}$, where $\left\{w^{j}\right\}_{j \geq 1}$ is the basis of eigenfunctions furnished in Lemma 3.4. It is easy to prove that the weak solution $\vec{y}$ of $(R)$ in $(0, T)$ can be obtained as a limit of the Galerkin approximations, $\vec{y}_{m}:[0, T] \rightarrow V_{m}$, solving the ordinary differential problem:

$$
(R)_{m}\left\{\begin{array}{l}
\frac{d}{d t} \int_{\Omega} \vec{y}_{m}(t) \cdot \vec{v}_{m} d \Omega+\nu_{h} \int_{\Omega} \nabla \vec{y}_{m}(t): \nabla \vec{v}_{m} d \Omega+\nu_{v} \int_{\Omega} \partial_{z} \vec{y}_{m}(t) \cdot \partial_{z} \vec{v}_{m} d \Omega \\
=\int_{\Omega} \vec{h}_{m}(t) \cdot \vec{v}_{m} d \Omega, \quad \forall v_{m} \in V_{m} \\
\vec{y}_{m}(0)=\vec{y}_{0 m}=\sum_{j=1}^{m}\left(\int_{\Omega} \nabla \vec{y}_{0}: \nabla \vec{w}^{j} d \Omega\right) \vec{w}^{j}
\end{array}\right.
$$

being $\vec{h}_{m}$ regular approximations to $\vec{h}$.

Now, we are going to obtain strong estimations for $\vec{y}_{m}$. First, by construction, $\vec{y}_{0_{m}} \in$ $V_{m}$ and $\vec{y}_{0 m} \rightarrow \vec{y}_{0}$ in $V$. Considering $\vec{v}_{m}=A \vec{y}_{m}(t) \in V_{m}$ as test functions in $(R)_{m}$, we obtain: $\forall t \in[0, T]$,

$$
\frac{d}{d t}\left\|\vec{y}_{m}(t)\right\|_{V}^{2}+\left\|A \vec{y}_{m}(t)\right\|_{L^{2}(\Omega)}^{2} \leq\left\|\vec{h}_{m}(t)\right\|_{L^{2}(\Omega)}^{2}
$$

Here and in the sequel, the notation $\|\vec{y}\|_{V}^{2}=\nu_{h}\|\nabla \vec{y}\|_{L^{2}(\Omega)}^{2}+\nu_{v}\left\|\partial_{z} \vec{y}\right\|_{L^{2}(\Omega)}^{2}$ will be used. Integrating in time:

$$
\left\|\vec{y}_{m}(t)\right\|_{V}^{2}+\int_{0}^{T}\left\|A \vec{y}_{m}(t)\right\|_{L^{2}(\Omega)}^{2} d t \leq\left\|\vec{y}_{0 m}\right\|_{V}^{2}+\int_{0}^{T}\left\|\vec{h}_{m}(t)\right\|_{L^{2}(\Omega)}^{2} d t .
$$

Then, the sequence $\left(\vec{y}_{m}\right)$ is bounded in $L^{2}(0, T ; D(A)) \cap L^{\infty}(0, T ; V)$, hence the limit verifies $\vec{y} \in L^{2}(0, T ; D(A)) \cap L^{\infty}(0, T ; V)$ and

$$
\|\vec{y}\|_{L^{\infty}(V)}^{2}+\|\vec{y}\|_{L^{2}(D(A))}^{2} \leq C\left\{\left\|\vec{y}_{0}\right\|_{V}^{2}+\|\vec{h}\|_{L^{2}\left(L^{2}(\Omega)\right)}^{2}\right\} .
$$

On the other hand, taking $\partial_{t} \vec{y}_{m}(t) \in V_{m}$ as test functions in $(R)_{m}$ and integrating in time,

$$
\left\|\partial_{t} \vec{y}_{m}\right\|_{L^{2}(H)}^{2} \leq\left\|\vec{y}_{0 m}\right\|_{V}^{2}+\left\|\vec{h}_{m}\right\|_{L^{2}\left(L^{2}(\Omega)\right)}^{2} .
$$

Therefore, the limit $\partial_{t} \vec{y} \in L^{2}(0, T ; H)$ and

$$
\left\|\partial_{t} \vec{y}\right\|_{L^{2}(H)}^{2} \leq C\left\{\left\|\vec{y}_{0}\right\|_{V}^{2}+\|\vec{h}\|_{L^{2}\left(L^{2}(\Omega)\right)}^{2}\right\} .
$$

Finally, from (21) and (22), using that $\vec{y}_{0}=\vec{v}_{0}-\vec{e}(0)$ and $\vec{h}=\vec{f}-\partial_{t} \vec{e}+\nu_{h} \Delta \vec{e}+\nu_{v} \partial_{z z}^{2} \vec{e}$,

$$
\begin{gathered}
\|\vec{y}\|_{L^{\infty}(V)}^{2}+\|\vec{y}\|_{L^{2}(D(A))}^{2}+\left\|\partial_{t} \vec{y}\right\|_{L^{2}(H)}^{2} \leq C\left\{\left\|\vec{v}_{0}\right\|_{V}^{2}+\|\vec{e}(0)\|_{V}^{2}\right. \\
\left.+\|\vec{f}\|_{L^{2}\left(L^{2}(\Omega)\right)}^{2}+\|\vec{e}\|_{L^{2}\left(H^{2}(\Omega)\right)}^{2}+\left\|\partial_{t} \vec{e}\right\|_{L^{2}\left(L^{2}(\Omega)\right)}^{2}\right\} .
\end{gathered}
$$

Using the above bounds of the $\vec{e}$ and $\vec{y},(17),(19),(20)$ and (23), we conclude the estimation (16). 


\section{Global strong solution for small data}

\subsection{Proof of Theorem 1.3}

We focus our study on the strong regularity of the problem $\left(E P_{2}\right)$. To do that, first we lift the nonhomogeneous Neumann boundary condition $\left.\nu_{v} \partial_{z} u\right|_{\Gamma_{s}}=\tau$, considering the lifting function $\left(e(t), q_{s}(t)\right)$, the strong solution of the two-dimensional version of problem $\left(L_{s t}\right)$, with $a=\tau$ and $f=0$ (this solution has already been used in Section 4). Then, it suffices to study the following problem that verifies $\left(w=u-e, \pi_{s}=p_{s}-q_{s}\right)$, being $\left(u, p_{s}\right)$ a solution of $\left(E P_{2}\right)$,

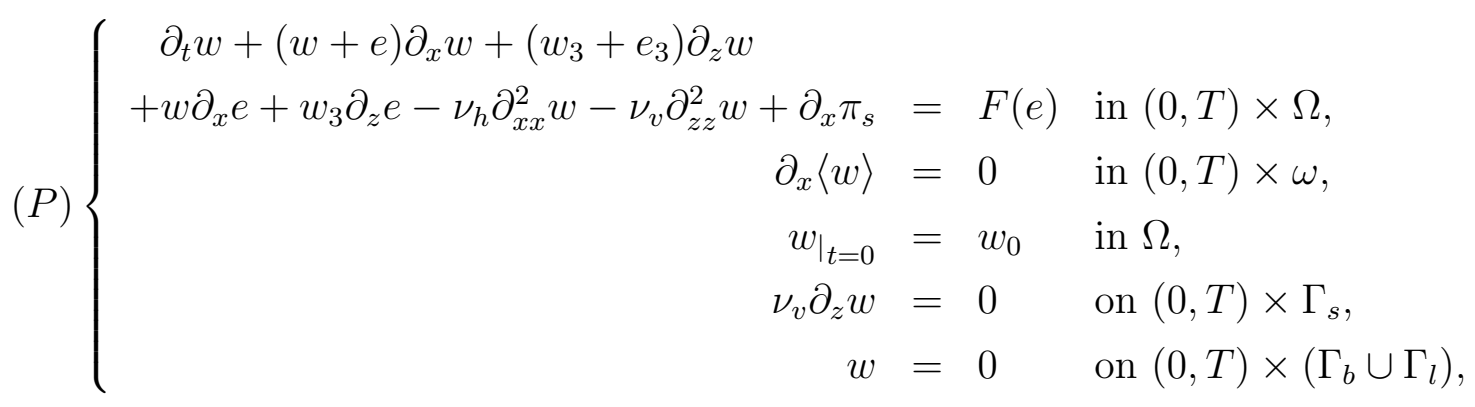

where $w_{0}=u_{0}-e(0)$ and $F(e)=F-\partial_{t} e-e \partial_{x} e-e_{3} \partial_{z} e$. We will study problem $(P)$ in several steps.

Step 1. Existence and weak estimates of the approximate solutions of $(P)$. We approximate $w$ by $w^{m}$, the Galerkin approximations in $V_{m}=\operatorname{span}\left\{w^{1}, \ldots, w^{m}\right\}$, being $\left\{w^{j}\right\}_{j \geq 1}$ the eigenfunctions of the hydrostatic Stokes operator $A$ (see Lemma 3.4). This is, $w_{m} \in C^{1}\left(0, T ; V_{m}\right)$ and satisfies the following variational formulation (in $V_{m}$ ), where $F_{m}$ denotes regular approximations to $F$,

$$
\left\{\begin{array}{l}
\int_{\Omega}\left(\frac{d}{d t} w_{m}+A w_{m}+\left(w_{m}+e\right) \partial_{x} w_{m}+\left(w_{m 3}+e_{3}\right) \partial_{z} w_{m}\right) v_{m} d \Omega \\
\quad+\int_{\Omega}\left(w_{m} \partial_{x} e+w_{m 3} \partial_{z} e\right) v_{m} d \Omega=\int_{\Omega}\left(F_{m}-e \partial_{x} e-e_{3} \partial_{z} e\right) v_{m} d \Omega \quad \forall v_{m} \in V_{m} \\
w_{m}(0)=w_{m 0}=\sum_{j=1}^{m}\left(\int_{\Omega} \nabla w_{0} \cdot \nabla w^{j} d \Omega\right) w^{j}
\end{array}\right.
$$

In the sequel, we denote $F_{m}=F$.

To prove existence of solution $w_{m}$ of (24), we write

$$
w_{m}(t ; x, z)=\sum_{j=1}^{m} \phi_{j}(t) w^{j}(x, z) .
$$

Introducing (25) in the expression (24) for $v_{m}=w^{i}, i=1, . ., m$, we conclude that $w_{m}$ is a solution of $(24)$ if only if $\left\{\phi_{j}\right\}_{j=1}^{m} \in C^{1}([0, T])^{m}$ and verifies the following ordinary 
differential problem:

$$
\left\{\begin{array}{l}
\sum_{j=1}^{m} a_{i j} \phi_{j}^{\prime}(t)+\sum_{j=1}^{m} b_{i j}(t) \phi_{j}(t)+\sum_{l, j=1}^{m} c_{i j l} \phi_{l}(t) \phi_{j}(t)=d_{i}(t) \text { en }(0, T), 1 \leq i \leq m, \\
\phi_{j}(0)=\int_{\Omega} \nabla u_{0} \cdot \nabla w^{j} d \Omega, \quad j=1, . ., m,
\end{array}\right.
$$

where the coefficients are:

$$
\begin{aligned}
a_{i j} & =\int_{\Omega} w^{j} w^{i} d \Omega, \\
b_{i j}(t) & =\int_{\Omega}\left\{\left(e(t) \partial_{x} w^{j}+e_{3}(t) \partial_{z} w^{j}+w^{j} \partial_{x} e(t)+w_{3}^{j} \partial_{z} e(t)\right) w^{i}\right. \\
& \left.+\nu_{h} \partial_{x} w^{j} \partial_{x} w^{i}+\nu_{v} \partial_{z} w^{j} \partial_{z} w^{i}\right\} d \Omega \in C^{0}([0, T]), \\
c_{i j l} & =\int_{\Omega}\left\{w^{l} \partial_{x} w^{j}+w_{3}^{l} \partial_{z} w^{j}\right\} w^{i} d \Omega, \\
d_{i}(t) & =\int_{\Omega}\left\{F(t)-e(t) \partial_{x} e(t)-e_{3}(t) \partial_{z} e(t)\right\} w^{i} d \Omega \in C^{0}([0, T]) .
\end{aligned}
$$

Using that $\left\{w^{j}\right\}$ is an orthonormal system in $H$, it is easy to prove that the matrix $\left(a_{i j}\right)_{i, j=1}^{m}$ is symmetric and definite positive (uniformely in $(0, T)$ ). In particular, matrix $\left(a_{i j}\right)_{i, j=1}^{m}$ is invertible and, therefore, (26) can be written as a Cauchy problem:

$$
(P C)\left\{\begin{array}{l}
\Phi^{\prime}=g(t, \Phi), \quad t \in[0, T] \\
\Phi(0) \in \mathbb{R}^{m} \text { given }
\end{array}\right.
$$

where $g: \mathbb{R}^{m+1} \rightarrow \mathbb{R}^{m}$ is a continuous function respect to $(t, \Phi)$, and smooth respect to $\Phi$ (in fact, $g(t, \cdot)$ is a polynomial function). By Picard's Theorem, one has existence and uniqueness of local solution $\Phi=\left(\phi_{i}\right)_{i=1}^{m}$, i.e. define in a interval $\left[0, \mathcal{T}_{m}\right)$ for some $\mathcal{T}_{m}>0$.

By uniqueness of the approximate problems for $u_{m}$ and $w_{m}$, one has $w_{m}=u_{m}-e$. Then, the weak estimations obtained for $u_{m}$ (see Remark 4.1) leads to the same weak estimations for $w_{m}$. In particular, taking into account expression (25),

$$
\sum_{j=1}^{m}\left|\phi_{j}(t)\right|^{2}=\int_{\Omega}\left|w_{m}\right|^{2} d \Omega \leq C \quad \forall t \in[0, T]
$$

Therefore, the positive semitrajectory of $\Phi,\left\{\left(s, \phi_{1}(s), . ., \phi_{m}(t)\right) \in \mathbb{R}^{m+1}, s \in\left[0, \mathcal{T}_{m}\right)\right\}$, is bounded in $\mathbb{R}^{m+1}$, so that $\Phi$ can be extended to all $[0, T]$. From this fact, we conclude existence of the approximate solution $w_{m}$ in all $[0, T]$ and their corresponding weak estimations.

Step 2. Strong estimates of the approximate solutions of $(P)$ : We recall the convection for the $V$-norm that follows:

$$
\|v\|_{V}^{2}=\nu_{h}\left\|\partial_{x} v\right\|_{L^{2}(\Omega)}^{2}+\nu_{v}\left\|\partial_{z} v\right\|_{L^{2}(\Omega)}^{2} .
$$


Lemma 5.1 The following estimate holds:

$$
\begin{aligned}
& \frac{d}{d t}\left\|w_{m}\right\|_{V}^{2}+\left\|A w_{m}\right\|_{L^{2}(\Omega)}^{2} \leq C_{1} D_{\max }\left\|A w_{m}\right\|_{L^{2}(\Omega)}^{2}\left\|w_{m}\right\|_{V}+C_{2}\left\|w_{m}\right\|_{V}^{4} \\
& +C_{3}\left\{\|e\|_{V}^{2}\|e\|_{L^{2}(\Omega)}^{2}+\left(D_{\max }^{4}+1\right)\|e\|_{H^{2}(\Omega)}^{2}\|e\|_{V}^{2}\right\}\left\|w_{m}\right\|_{V}^{2} \\
& +C_{4}\|e\|_{H^{2}(\Omega)}\|e\|_{V}^{2}\left(\|e\|_{L^{2}(\Omega)}+D_{\max }^{2}\|e\|_{H^{2}(\Omega)}\right) \\
& +C_{5}\|F\|_{L^{2}(\Omega)}^{2}+C_{6}\left\|\partial_{t} e\right\|_{L^{2}(\Omega)}^{2}
\end{aligned}
$$

for some $C_{i}>0, i=1, . ., 6$.

Proof: Taking $v_{m}=A w_{m}(t) \in V_{m}$ as test functions in (24) (here, we have used the properties of the $A$-eigenfunction basis), one has:

$$
\begin{aligned}
& \frac{1}{2} \frac{d}{d t}\left\|w_{m}\right\|_{V}^{2}+\left\|A w_{m}\right\|_{L^{2}(\Omega)}^{2}=-\int_{\Omega}\left(w_{m}+e\right) \partial_{x} w_{m} A w_{m} d \Omega \\
& -\int_{\Omega}\left(w_{m 3}+e_{3}\right) \partial_{z} w_{m} A w_{m} d \Omega-\int_{\Omega} w_{m} \partial_{x} e A w_{m} d \Omega \\
& -\int_{\Omega} w_{m 3} \partial_{z} e A w_{m} d \Omega+\int_{\Omega} F(e) A w_{m} d \Omega \equiv \sum_{i=1}^{5} I_{i}
\end{aligned}
$$

We bound the right hand side (using Lemmas 2.1, 2.2, 2.3 and 3.3, and the weak estimates for $w_{m}$ ):

$$
\begin{aligned}
& I_{1} \leq\left\|A w_{m}\right\|_{L^{2}(\Omega)}\left\|\partial_{x} w_{m}\right\|_{L^{4}(\Omega)}\left(\left\|w_{m}\right\|_{L^{4}(\Omega)}+\|e\|_{L^{4}(\Omega)}\right) \\
& \leq C\left\|A w_{m}\right\|_{L^{2}(\Omega)}^{3 / 2}\left\|\partial_{x} w_{m}\right\|_{L^{2}(\Omega)}^{1 / 2}\left(\left\|\nabla w_{m}\right\|_{L^{2}(\Omega)}^{1 / 2}\left\|w_{m}\right\|_{L^{2}(\Omega)}^{1 / 2}+\|\nabla e\|_{L^{2}(\Omega)}^{1 / 2}\|e\|_{L^{2}(\Omega)}^{1 / 2}\right) \\
& \leq \frac{1}{10}\left\|A w_{m}\right\|_{L^{2}(\Omega)}^{2}+C\left\|w_{m}\right\|_{V}^{2}\left(\left\|w_{m}\right\|_{V}^{2}+\|e\|_{V}^{2}\|e\|_{L^{2}(\Omega)}^{2}\right) \\
& I_{2} \leq\left\|A w_{m}\right\|_{L^{2}(\Omega)}\left\|\partial_{z} w_{m}\right\|_{L^{4}(\Omega)}\left(\left\|w_{m_{3}}\right\|_{L^{4}(\Omega)}+\left\|e_{3}\right\|_{L^{4}(\Omega)}\right) \\
& \leq D_{\max }\left\|A w_{m}\right\|_{L^{2}(\Omega)}^{3 / 2}\left\|\partial_{z} w_{m}\right\|_{L^{2}(\Omega)}^{1 / 2}\left(\left\|\partial_{x} w_{m}\right\|_{L^{4}(\Omega)}+\left\|\partial_{x} e\right\|_{L^{4}(\Omega)}\right) \\
& \leq C D_{\max }\left\|A w_{m}\right\|_{L^{2}(\Omega)}^{3 / 2}\left\|w_{m}\right\|_{V}^{1 / 2}\left(\left\|A w_{m}\right\|_{L^{2}(\Omega)}^{1 / 2}\left\|w_{m}\right\|_{V}^{1 / 2}+\|e\|_{H^{2}(\Omega)}^{1 / 2}\|e\|_{V}^{1 / 2}\right) \\
& \leq C D_{\max }\left\|A w_{m}\right\|_{L^{2}(\Omega)}^{2}\left\|w_{m}\right\|_{V}+\frac{1}{10}\left\|A w_{m}\right\|_{L^{2}(\Omega)}^{2}+C D_{m a x}^{4}\left\|w_{m}\right\|_{V}^{2}\|e\|_{H^{2}(\Omega)}^{2}\|e\|_{V}^{2} \\
& \leq\left\|w_{m}\right\|_{L^{4}(\Omega)}\left\|\partial_{x} e\right\|_{L^{4}(\Omega)}\left\|A w_{m}\right\|_{L^{2}(\Omega)} \\
& \leq C\left\|A w_{m}\right\|_{L^{2}(\Omega)}\left\|w_{m}\right\|_{V}^{1 / 2}\left\|w_{m}\right\|_{L^{2}(\Omega)}^{1 / 2}\|e\|_{H^{2}(\Omega)}^{1 / 2}\|e\|_{V}^{1 / 2} \\
& \leq \frac{1}{10}\left\|A w_{m}\right\|_{L^{2}(\Omega)}^{2}+C\left\|w_{m}\right\|_{V}^{2}\|e\|_{H^{2}(\Omega)}^{2}\|e\|_{V}^{2}
\end{aligned}
$$




$$
\begin{aligned}
& I_{4} \leq\left\|w_{m 3}\right\|_{L^{4}(\Omega)}\left\|\partial_{z} e\right\|_{L^{4}(\Omega)}\left\|A w_{m}\right\|_{L^{2}(\Omega)} \\
& \leq D_{\text {max }}\left\|\partial_{x} w_{m}\right\|_{L^{4}(\Omega)}\left\|\partial_{z} e\right\|_{L^{4}(\Omega)}\left\|A w_{m}\right\|_{L^{2}(\Omega)} \\
& \leq C D_{\max }\left\|A w_{m}\right\|_{L^{2}(\Omega)}^{3 / 2}\left\|w_{m}\right\|_{V}^{1 / 2}\|e\|_{H^{2}(\Omega)}^{1 / 2}\|e\|_{V}^{1 / 2} \\
& \leq \frac{1}{10}\left\|A w_{m}\right\|_{L^{2}(\Omega)}^{2}+C D_{\text {max }}^{4}\left\|w_{m}\right\|_{V}^{2}\|e\|_{H^{2}(\Omega)}^{2}\|e\|_{V}^{2} \\
& I_{5} \leq\left\|A w_{m}\right\|_{L^{2}(\Omega)}\|F(e)\|_{L^{2}(\Omega)} \leq \frac{1}{10}\left\|A w_{m}\right\|_{L^{2}(\Omega)}^{2}+\frac{5}{2}\|F(e)\|_{L^{2}(\Omega)}^{2}
\end{aligned}
$$

To bound the $\|F(e)\|_{L^{2}(\Omega)}^{2}$ term, we write:

$$
\frac{5}{2}\|F(e)\|_{L^{2}(\Omega)}^{2} \leq C\left\{\|F\|_{L^{2}(\Omega)}^{2}+\left\|\partial_{t} e\right\|_{L^{2}(\Omega)}^{2}+\left\|e \partial_{x} e\right\|_{L^{2}(\Omega)}^{2}+\left\|e_{3} \partial_{z} e\right\|_{L^{2}(\Omega)}^{2}\right\} \equiv \sum_{i=1}^{4} J_{i}
$$

where:

$$
\begin{aligned}
J_{3} & \leq\left\|\partial_{x} e\right\|_{L^{4}(\Omega)}^{2}\|e\|_{L^{4}(\Omega)}^{2} \leq C\|e\|_{H^{2}(\Omega)}\|e\|_{V}^{2}\|e\|_{L^{2}(\Omega)} \\
J_{4} & \leq\left\|e_{3}\right\|_{L^{4}(\Omega)}^{2}\left\|\partial_{z} e\right\|_{L^{4}(\Omega)}^{2} \leq D_{\max }^{2}\left\|\partial_{x} e\right\|_{L^{4}(\Omega)}^{2}\left\|\partial_{z} e\right\|_{L^{4}(\Omega)}^{2} \\
& \leq C D_{\text {max }}^{2}\|e\|_{H^{2}(\Omega)}^{2}\|e\|_{V}^{2}
\end{aligned}
$$

Incorporing all these bounds in (28), we have (27).

The most difficult term in (27) (to bound globally in time) is $C_{1} D_{\max }\left\|A w_{m}\right\|_{L^{2}(\Omega)}^{2}\left\|w_{m}\right\|_{V}$. One possibility is to control this one with the $\left\|A w_{m}\right\|_{L^{2}(\Omega)}^{2}$ term of the left hand side of (27), when small data are assumed.

We rewrite (27) in the form:

$$
\begin{array}{r}
\frac{d}{d t}\left\|w_{m}\right\|_{V}^{2}+ \\
\leq C_{2}\left\|w_{m}\right\|_{L^{2}(\Omega)}^{2}\left(1-C_{1} D_{\max }^{4}\left\|w_{m}\right\|_{V}\right) \\
\leq a(t)\left\|w_{m}\right\|_{V}^{2}+b(t),
\end{array}
$$

where:

$$
\begin{aligned}
a(t) & =C_{3}\left\{\|e\|_{V}^{2}\|e\|_{L^{2}(\Omega)}^{2}+\left(D_{\text {max }}^{4}+1\right)\|e\|_{H^{2}(\Omega)}^{2}\|e\|_{V}^{2}\right\} \\
b(t) & =C_{4}\|e\|_{H^{2}(\Omega)}\|e\|_{V}^{2}\left(\|e\|_{L^{2}(\Omega)}+D_{\text {max }}^{2}\|e\|_{H^{2}(\Omega)}\right) \\
& +C_{5}\|F\|_{L^{2}(\Omega)}^{2}+C_{6}\left\|\partial_{t} e\right\|_{L^{2}(\Omega)}^{2} .
\end{aligned}
$$

It is easy to check that $a, b \in L^{1}(0, T)$. Indeed, using the estimates for $e(17),(19)$ and (20), one has

$$
\begin{aligned}
\|a\|_{L^{1}(0, T)} & \leq C_{3}\left(D_{\max }^{4}+1\right) A(\tau)^{2} \\
\|b\|_{L^{1}(0, T)} & \leq\left\{C_{4}\left(D_{\max }^{2}+1\right)+C_{6}\right\} A(\tau)^{2}+C_{5}\|F\|_{L^{2}\left(L^{2}(\Omega)\right)}^{2},
\end{aligned}
$$

where $A(\tau)^{2}=C^{2}\left(\|\tau(0)\|_{H^{-1 / 2}\left(\Gamma_{s}\right)}^{2}+\|\tau\|_{L^{2}\left(H_{0}^{1 / 2+\varepsilon}\left(\Gamma_{s}\right)\right)}^{2}+\left\|\partial_{t} \tau\right\|_{L^{2}\left(H^{-1 / 2}\left(\Gamma_{s}\right)\right)}^{2}\right)$. 
Lemma 5.2 Under the hypothesis $(H)$ of the Theorem 1.3 and supposing (29), let $M$ be a constant such that :

(a) $\quad 1-C_{1} D_{\max } M>1 / 2$,

(b) $\quad C_{2} M^{2}<1 /(4 C)$,

$\left(C_{1}\right.$ and $C_{2}$ are the constants that appear in (29) and $C>0$ is the equivalence constant between $\|A u\|_{L^{2}}$ and the $H^{2}$-norm, see Lemma 3.3), then

$$
\left\|w_{m}(t)\right\|_{V}<M, \quad \forall t \in[0, T] .
$$

Proof: Arguing by contradiction, we suppose there exists some instant in $(0, T)$ where the bound $M$ is reached. Let $t^{*}$ the smallest of these instants, i.e. $\left\|w_{m}(t)\right\|_{V}<M$, $\forall t \in\left[0, t^{*}\right)$ and $\left\|w_{m}\left(t^{*}\right)\right\|_{V}=M$. Then, $\forall t \in\left[0, t^{*}\right]$,

$$
1-C_{1} D_{\max }\left\|w_{m}(t)\right\|_{V} \geq 1-C_{1} D_{\max } M>1 / 2 .
$$

In the last estimation, we have used hypothesis $(a)$. If we denote $y(t)=\left\|w_{m}(t)\right\|_{V}^{2}$, using that $\frac{1}{C}\left\|w_{m}\right\|_{V}^{2} \leq\left\|A w_{m}\right\|_{L^{2}(\Omega)}^{2}$ (see (15) in Lemma 3.3), (29) yields:

$$
y^{\prime}(t)+\frac{1}{2 C} y(t) \leq C_{2} M^{2} y(t)+a(t) y(t)+b(t), \quad \forall t \in\left[0, t^{*}\right] .
$$

Now, from hypothesis $(b)$,

$$
y^{\prime}(t)+\frac{1}{4 C} y(t) \leq a(t) y(t)+b(t), \quad \forall t \in\left[0, t^{*}\right] .
$$

Integrating this differential inequality between 0 and $t^{*}$, we obtain:

$$
y\left(t^{*}\right) \leq \exp \left(-\frac{1}{4 C} t^{*}+\int_{0}^{t^{*}} a(t) d t\right)\left\{y(0)+\int_{0}^{t^{*}} \exp \left(\frac{1}{4 C} t-\int_{0}^{t} a(s) d s\right) b(t) d t\right\}
$$

Therefore, since

$$
y(0)=\left\|w_{m 0}\right\|_{V}^{2} \leq\left\|w_{0}\right\|_{V}^{2} \leq 2\left(\left\|u_{0}\right\|_{V}^{2}+\|e(0)\|_{V}^{2}\right) \leq 2\left(\left\|u_{0}\right\|_{V}^{2}+C^{2}\|\tau(0)\|_{H^{-1 / 2}\left(\Gamma_{s}\right)}^{2}\right),
$$

hypothesis $(H)$ implies $\left\|w_{m}\left(t^{*}\right)\right\|_{V}<M$, hence we arrive at contradiction.

Step 3. Proof of Theorem 1.3: From Lemma 5.2, $w_{m}$ is bounded in $L^{\infty}(0, T ; V)$. Moreover, applying hypothesis $(a)$ of Lemma 5.2 in (29), one has

$$
\frac{d}{d t}\left\|w_{m}\right\|_{V}^{2}+\frac{1}{2}\left\|A w_{m}\right\|_{L^{2}(\Omega)}^{2} \leq C_{2} M^{4}+M^{2} a(t)+b(t),
$$

hence, integrating in time, we deduce that $w_{m}$ is bounded in $L^{2}\left(0, T ; H^{2}(\Omega)\right)$. On the other hand, taking $\partial_{t} w_{m}(t) \in V_{m}$ as a test function in (24), integrating in time and using the above regularity, one deduces that $\partial_{t} w_{m}$ is bounded in $L^{2}(0, T ; H)$. Then, by a standard argument of passage to the limit, we obtain that $w$ (and a surface pressure associated $\pi_{s}$ ) is a strong global solution of $(P)$. Finally, $\left(u, p_{s}\right)=\left(e+w, q_{s}+\pi_{s}\right)$ is a strong solution of $\left(E P_{2}\right)$ in $(0, T)$. The uniqueness of strong solution of $\left(E P_{2}\right)$ stems from Section 7. 
Remark 5.1 In the 3D case, we cannot obtain the above strong estimates. It is because in the right hand side of (27), if we estimate the corresponding $I_{2}$ term, we obtain a bound of the form

$$
D_{\max }\left\|A w_{m}\right\|_{L^{2}(\Omega)}^{5 / 2}\left\|w_{m}\right\|_{V}^{1 / 2}
$$

which cannot be controlated with the left hand side of (27).

\subsection{Proof of Corollary 1.4.}

Let us first prove existence of a strong solution of $\left(E P_{2}\right)$ in $(0,+\infty)$. The argument is based in Step 1 and 2 of the proof of Theorem 1.3. In particular, it is not difficult to obtain the global weak estimations:

$$
w_{m} \text { is bounded in } L^{2}(0,+\infty ; V) \cap L^{\infty}(0,+\infty ; H) .
$$

Now, using hypothesis $(H)$ in $[0,+\infty)$, we can deduce that $\left\|w_{m}\right\|_{V}<M, \forall t \in[0+\infty)$.

Let us change Step 3. Instead of (33), we rewrite (29) as:

$$
\frac{d}{d t}\left\|w_{m}\right\|_{V}^{2}+\frac{1}{2}\left\|A w_{m}\right\|_{L^{2}(\Omega)}^{2} \leq C_{2} M^{2}\left\|w_{m}\right\|_{V}^{2}+M^{2} a(t)+b(t) .
$$

Using that $w_{m}$ is bounded in $L^{2}(0,+\infty ; V)$ and $a, b \in L^{1}(0,+\infty)$ (thanks to (31) and the global regularity of $\tau, \partial_{t} \tau$ and $\left.F\right)$, we have that $w_{m}$ is bounded in $L^{2}\left(0,+\infty ; H^{2}(\Omega) \cap\right.$ $V)$. Then, we can conclude the existence of a strong solution $u \in L^{\infty}(0,+\infty ; V) \cap$ $L^{2}\left(0,+\infty ; H^{2}(\Omega) \cap V\right)$ and $\partial_{t} u \in L^{2}(0,+\infty ; H)$.

Now, let us see the asymptotic behaviour of $u$. Adding in both parts of (32) $\frac{d}{d t}\|e(t)\|_{V}^{2}+$ $\frac{1}{4 C}\|e(t)\|_{V}^{2}$, taking into account that

$$
\frac{d}{d t}\|e(t)\|_{V}^{2} \leq 2\|e(t)\|_{V}\left\|\partial_{t} e(t)\right\|_{V}
$$

we obtain for $z(t)=\left\|w_{m}(t)\right\|_{V}^{2}+\|e(t)\|_{V}^{2}$ the inequality:

$$
z^{\prime}(t)+\left(\frac{1}{4 C}-a(t)\right) z(t) \leq b(t)+\frac{1}{2 C}\|e(t)\|_{V}^{2}+4 C\left\|\partial_{t} e(t)\right\|_{V}^{2}
$$

Multiplying by $\exp \left(\frac{1}{4 C} t-\int_{0}^{t} a(s) d s\right)$ and integrating on $(0, t)$,

$$
\begin{aligned}
z(t) & \leq \exp \left(-\frac{1}{4 C} t+\int_{0}^{t} a(s) d s\right)\{z(0) \\
& \left.+\int_{0}^{t} \exp \left(\frac{1}{4 C} s-\int_{0}^{s} a(\sigma) d \sigma\right)\left(b(s)+\frac{1}{2 C}\|e(s)\|_{V}^{2}+4 C\left\|\partial_{t} e(s)\right\|_{V}^{2}\right) d s\right\} .
\end{aligned}
$$

Now, using that $\left\|u_{m}(t)\right\|_{V}^{2} \leq 2 z(t)$,

$$
\begin{aligned}
\left\|u_{m}(t)\right\|_{V}^{2} & \leq \exp \left(-\frac{1}{4 C} t\right) K_{1}\{z(0) \\
& \left.+\frac{2}{K_{1}} \int_{0}^{t} \exp \left(\frac{1}{4 C} s\right)\left(b(s)+\frac{1}{2 C}\|e(s)\|_{V}^{2}+4 C\left\|\partial_{t} e(s)\right\|_{V}^{2}\right) d s\right\},
\end{aligned}
$$


where $K_{1}=2 \exp \left(\|a\|_{L^{1}(0,+\infty)}\right)$. Since $z(0) \leq 2\left\|u_{0}\right\|_{V}^{2}+C^{2}\|\tau(0)\|_{H^{-1 / 2}\left(\Gamma_{s}\right)}^{2}$, bounding in a convenient way $b, e$ and $\partial_{t} e$ (in function of $\tau, \partial_{t} \tau$ and $F$ ), we can deduce the asymptotic behaviour (5) whenever the hypothesis (4) holds.

\section{Local strong solution for any data (proof of Theo- rem 1.4)}

We want to apply now a fixed point argument to obtain strong solution of $\left(E P_{2}\right)$, local in time, but for any data. Now, we study problem $(Q)$, which is similar to $(P)$ but whose solution is $\left(w=u-v, \tilde{\pi}_{s}=p_{s}-q_{s}\right)$, where $\left(v, q_{s}\right)$ is the solution of $(L)$ with $v_{0}=0$ anf $f=0$. With this purpose, we rewrite $(Q)$ as a fixed point equation by means of a linearisation. We define, for each $T>0$ :

$$
\begin{array}{r}
Y(T)=\left\{\bar{w} ; \bar{w} \in L^{2}(0, T ; D(A)) \cap L^{\infty}(0, T ; V), \partial_{t} \bar{w} \in L^{2}(0, T ; H),\right. \\
\left.\bar{w}(0)=u_{0},\|\bar{w}\|_{L^{\infty}(V)}^{2}+\|\bar{w}\|_{L^{2}(D(A))}^{2}+\left\|\partial_{t} \bar{w}\right\|_{L^{2}(H)}^{2} \leq R^{2}\right\} .
\end{array}
$$

Given $v$ the strong solution of $(L)$ in $(0, T)$ and $\bar{w} \in Y(T)$, we consider the linear problem:

$$
\left(Q_{l}\right)\left\{\begin{aligned}
\partial_{t} w-\nu_{h} \partial_{x x}^{2} w-\nu_{v} \partial_{z z}^{2} w+\partial_{x} \pi_{s} & =G(\bar{w}, v) & & \text { in }(0, T) \times \Omega, \\
\partial_{x}\langle w\rangle & =0 & & \text { in }(0, T) \times \omega, \\
w_{t=0} & =u_{0} & & \text { in } \Omega, \\
\nu_{v} \partial_{z} w & =0 & & \text { on }(0, T) \times \Gamma_{s}, \\
w & =0 & & \text { on }(0, T) \times\left(\Gamma_{b} \cup \Gamma_{l}\right),
\end{aligned}\right.
$$

where $G(\bar{w}, v)=F-(\bar{w}+v) \partial_{x}(\bar{w}+v)-\left(\bar{w}_{3}+v_{3}\right) \partial_{z}(\bar{w}+v)$. Problem $\left(Q_{l}\right)$ is similar to problem $(R)$, which has already been studied in Section 4 . Therefore, since $u_{0} \in V$ and $G \in L^{2}((0, T) \times \Omega)$, then $w \in L^{2}(0, T ; D(A)) \cap L^{\infty}(0, T ; V)$ and $\partial_{t} w \in L^{2}(0, T ; H)$.

First, we are going to prove that, there exists $R^{2}$ large enough such that $Y(T) \neq \emptyset$, $\forall T>0$. Indeed, let $w_{*}$ be the unique solution of the hydrostatic Stokes problem:

$$
\left\{\begin{aligned}
\partial_{t} w_{*}-\nu_{h} \partial_{x x}^{2} w_{*}-\nu_{v} \partial_{z z}^{2} w_{*}+\partial_{x} \pi_{s}=0 & \text { in }(0, T) \times \Omega, \\
\partial_{x}\left\langle w_{*}\right\rangle=0 & \text { in }(0, T) \times \omega, \\
w_{* \mid t=0}=u_{0} & \text { in } \Omega, \\
\nu_{v} \partial_{z} w_{*}=0 & \text { on }(0, T) \times \Gamma_{s}, \\
w_{*}=0 & \text { on }(0, T) \times\left(\Gamma_{b} \cup \Gamma_{l}\right) .
\end{aligned}\right.
$$

Following the reasoning of the problem $(R)$, see $(21)$ and (22), we know that:

$$
\left\|w_{*}\right\|_{L^{\infty}(V)}^{2}+\left\|w_{*}\right\|_{L^{2}(D(A))}^{2}+\left\|\partial_{t} w_{*}\right\|_{L^{2}(H)}^{2} \leq\left\|u_{0}\right\|_{V}^{2}
$$

therefore, taking $R^{2} \geq\left\|u_{0}\right\|_{V}^{2}$, then $w_{*} \in Y(T), \forall T>0$. 
Now, we introduce the Banach space $X_{T}=L^{2}(0, T ; V)$ and the mapping

$\Phi: Y(T) \longrightarrow X_{T}$, given by $\Phi(\bar{w})=w$,

where $w$ is the unique solution of $\left(Q_{l}\right)$. Obviously, a fixed point of $\Phi$ solves problem $(Q)$. Arguing as in problem $(R)$, we have:

$$
\|w\|_{L^{\infty}(V)}^{2}+\|w\|_{L^{2}(D(A))}^{2}+\left\|\partial_{t} w\right\|_{L^{2}(H)}^{2} \leq\left\|u_{0}\right\|_{V}^{2}+C\|G(\bar{w}, v)\|_{L^{2}\left(L^{2}(\Omega)\right)}^{2}
$$

On the other hand, $v$ verifies problem $(L)$, with inicial data zero and homogeneous second member (i.e. $v_{0}=0$ and $f=0$ ) but a nonhomogeneous Neumann boundary condition $(\tau)$ on the surface. Then, $v$ satisfies the estimate (see (16)):

$$
\|v\|_{L^{\infty}(V)}^{2}+\|v\|_{L^{2}\left(H^{2}(\Omega)\right)}^{2}+\left\|\partial_{t} v\right\|_{L^{2}(H)}^{2} \leq B(\tau)^{2},
$$

where $B(\tau)^{2}=C\left\{\|\tau(0)\|_{H^{-1 / 2}\left(\Gamma_{s}\right)}^{2}+\|\tau\|_{L^{2}\left(H_{0}^{1 / 2+\varepsilon}\left(\Gamma_{s}\right)\right)}^{2}+\left\|\partial_{t} \tau\right\|_{L^{2}\left(H^{-1 / 2}\left(\Gamma_{s}\right)\right)}^{2}\right\}$.

Now, we want to find conditions to apply Schauder's Theorem.

1) $\exists T_{*} \in(0, T]$ such that $\Phi\left(Y\left(T_{*}\right)\right) \subset Y\left(T_{*}\right)$ :

Let $\bar{w} \in Y(T)$ and $w=\Phi(\bar{w})$. Then:

$$
\begin{aligned}
\|G(\bar{w}, v)\|_{L^{2}\left(L^{2}(\Omega)\right)}^{2} & \leq 9\left\{\|F\|_{L^{2}\left(L^{2}(\Omega)\right)}^{2}+\left\|(\bar{w}+v) \partial_{x}(\bar{w}+v)\right\|_{L^{2}\left(L^{2}(\Omega)\right)}^{2}\right. \\
& \left.+\left\|\left(\bar{w}_{3}+v_{3}\right) \partial_{z}(\bar{w}+v)\right\|_{L^{2}\left(L^{2}(\Omega)\right)}^{2}\right\} \equiv \sum_{i=1}^{3} I_{i}
\end{aligned}
$$

We bound each term $I_{i}$ (constant $G$ will come from the Gagliardo-Nirenberg's inequalities, see Lemmas 2.2 and 2.3, whereas $C$ we will denote different constants independent of $R, B(\tau), D_{\max }$ and $\left.T\right)$. First, we estimate

$$
\begin{aligned}
\|(\bar{w} & +v) \partial_{x}(\bar{w}+v)\left\|_{L^{2}(\Omega)}^{2} \leq\right\| \bar{w}+v\left\|_{L^{4}(\Omega)}^{2}\right\| \partial_{x}(\bar{w}+v) \|_{L^{4}(\Omega)}^{2} \\
& \leq 4\left(\|\bar{w}\|_{L^{4}(\Omega)}^{2}+\|v\|_{L^{4}(\Omega)}^{2}\right)\left(\left\|\partial_{x} \bar{w}\right\|_{L^{4}(\Omega)}^{2}+\left\|\partial_{x} v\right\|_{L^{4}(\Omega)}^{2}\right) \\
& \leq 4 G^{2}\left(\|\bar{w}\|_{V}\|\bar{w}\|_{L^{2}(\Omega)}+\|v\|_{V}\|v\|_{L^{2}(\Omega)}\right)\left(\|\bar{w}\|_{H^{2}(\Omega)}\|\bar{w}\|_{V}+\|v\|_{H^{2}(\Omega)}\|v\|_{V}\right) .
\end{aligned}
$$

Integrating in $(0, T)$, taking into account definition of $Y(T)$ and $(37)$,

$$
\begin{aligned}
I_{2} & \leq 4 G^{2} T^{1 / 2}\left(\|\bar{w}\|_{L^{\infty}(V)}\|\bar{w}\|_{L^{\infty}(H)}+\|v\|_{L^{\infty}(V)}\|v\|_{L^{\infty}(H)}\right) \\
& \times\left(\|\bar{w}\|_{L^{\infty}(V)}\|\bar{w}\|_{L^{2}\left(H^{2}(\Omega)\right)}+\|v\|_{L^{\infty}(V)}\|v\|_{L^{2}\left(H^{2}(\Omega)\right)}\right) \\
& \leq C T^{1 / 2}\left(B(\tau)^{2}+R^{2}\right)^{2} .
\end{aligned}
$$

In a similar way, we bound the vertical velocity terms as follows:

$$
\begin{aligned}
\|\left(\bar{w}_{3}\right. & \left.+v_{3}\right) \partial_{z}(\bar{w}+v)\left\|_{L^{2}(\Omega)}^{2} \leq\right\| \bar{w}_{3}+v_{3}\left\|_{L^{4}(\Omega)}^{2}\right\| \partial_{z}(\bar{w}+v) \|_{L^{4}(\Omega)}^{2} \\
& \leq 4\left(\left\|\bar{w}_{3}\right\|_{L^{4}(\Omega)}^{2}+\left\|v_{3}\right\|_{L^{4}(\Omega)}^{2}\right)\left(\left\|\partial_{z} \bar{w}\right\|_{L^{4}(\Omega)}^{2}+\left\|\partial_{z} v\right\|_{L^{4}(\Omega)}^{2}\right) \\
& \leq 4 D_{\max }^{2}\left(\left\|\partial_{x} \bar{w}\right\|_{L^{4}(\Omega)}^{2}+\left\|\partial_{x} v\right\|_{L^{4}(\Omega)}^{2}\right)\left(\left\|\partial_{z} \bar{w}\right\|_{L^{4}(\Omega)}^{2}+\left\|\partial_{z} v\right\|_{L^{4}(\Omega)}^{2}\right) \\
& \leq 4 G^{2} D_{\max }^{2}\left(\|\bar{w}\|_{H^{2}(\Omega)}\|\bar{w}\|_{V}+\|v\|_{H^{2}(\Omega)}\|v\|_{V}\right)^{2} .
\end{aligned}
$$


Therefore, integrating in $(0, T)$,

$$
\begin{aligned}
I_{3} & \leq 4 C D_{\max }^{2}\left(\|\bar{w}\|_{L^{2}\left(H^{2}(\Omega)\right)}\|\bar{w}\|_{L^{\infty}(V)}+\|v\|_{L^{2}\left(H^{2}(\Omega)\right)}\|v\|_{L^{\infty}(V)}\right)^{2} \\
& \leq C D_{\max }^{2}\left(B(\tau)^{2}+R^{2}\right)^{2} .
\end{aligned}
$$

In the last estimates, we could not obtain any power of $T$, and this fact is the main difficulty in our argument. Indeed, inserting all the above bound in (38),

$$
\|G(\bar{w}, v)\|_{L^{2}\left(L^{2}(\Omega)\right)}^{2} \leq C\left\{\|F\|_{L^{2}\left(L^{2}(\Omega)\right)}^{2}+\left(B(\tau)^{2}+R^{2}\right)^{2}\left(D_{\max }^{2}+T^{1 / 2}\right)\right\}
$$

Then, from (36) and (39),

$$
\begin{aligned}
\|w\|_{L^{\infty}(V)}^{2} & +\|w\|_{L^{2}(D(A))}^{2}+\left\|\partial_{t} w\right\|_{L^{2}(H)}^{2} \leq\left\|u_{0}\right\|_{V}^{2} \\
& +C\left\{\|F\|_{L^{2}\left(L^{2}(\Omega)\right)}^{2}+\left(B(\tau)^{2}+R^{2}\right)^{2}\left(D_{\text {max }}^{2}+T^{1 / 2}\right)\right\}
\end{aligned}
$$

The above inequality can be written as

$$
\|w\|_{L^{\infty}(V)}^{2}+\|w\|_{L^{2}\left(H^{2}(\Omega)\right)}^{2}+\left\|\partial_{t} w\right\|_{L^{2}(H)}^{2} \leq a R^{4}+b R^{2}+c,
$$

where, for some $C=C\left(\Omega, \nu_{h}, \nu_{v}\right)>0$,

$$
\begin{aligned}
a & =C\left(D_{\max }^{2}+T^{1 / 2}\right) \\
b & =2 C B(\tau)^{2}\left(D_{\max }^{2}+T^{1 / 2}\right), \\
c & =\left\|u_{0}\right\|_{V}^{2}+C\left\{\|F\|_{L^{2}\left(0, T ; L^{2}(\Omega)\right)}^{2}+B(\tau)^{4}\left(D_{\max }^{2}+T^{1 / 2}\right)\right\} .
\end{aligned}
$$

Taking $R^{2} \geq\left\|u_{0}\right\|_{V}^{2}$ (hence $Y(T) \neq \emptyset, \forall T>0$ ), one has $w \in Y(T)$ whenever

$$
a R^{4}+b R^{2}+c \leq R^{2} .
$$

In the following, we will see that for any data $F, \tau, u_{0},(41)$ is verified. A necessary condition to (41) is $b<1$. But, it can also find some sufficient conditions. Indeed, one possibility is to impose the following three conditions:

Condition 1: $D_{\max }$ and $T$ are small enough sucht that

$$
b \leq \frac{1}{2}
$$

For instance, $2 C B(\tau)^{2} D_{\max }^{2} \leq 1 / 4$ and $2 C B(\tau)^{2} T^{1 / 2} \leq 1 / 4$.

Condition 2: $R^{2}$ big enough such that

$$
c \leq \frac{1}{4} R^{2}
$$

Condition 3: $a$ small enough (i.e. $D_{\max }$ and $T$ small enough) such that

$$
a R^{2} \leq \frac{1}{4}
$$


In conclusion, there exists $T_{*} \in(0, T]$ and $D_{\max }>0$ small enough, such that for some $R$ big enough, one has $\Phi\left(Y\left(T_{*}\right)\right) \subset Y\left(T_{*}\right)$.

\section{2) $Y\left(T_{*}\right)$ is relatively compact in $X_{T_{*}}$.}

Let $W_{T_{*}}=\left\{\bar{w} ; \bar{w} \in L^{2}\left(0, T_{*} ; D(A)\right)\right.$ and $\left.\partial_{t} \bar{w} \in L^{2}\left(0, T_{*} ; H\right)\right\} . Y\left(T_{*}\right)$ is a bounded set of $W_{T_{*}}$ and $W_{T_{*}}$ is embedded in a compact way in $X_{T_{*}}$. Therefore, $Y\left(T_{*}\right)$ is relatively compact in $X_{T_{*}}$.

3) $Y\left(T_{*}\right)$ is closed in $X_{T_{*}}$.

Let $\left\{\bar{w}_{n}\right\}_{n \geq 1} \subseteq Y\left(T_{*}\right)$ such that $\bar{w}_{n} \longrightarrow \bar{w}$ strongly in $X_{T_{*}}$ (i.e. in the $L^{2}(0, T ; V)$ norm). Let us see that $\bar{w} \in Y\left(T_{*}\right)$. As $\left\{\bar{w}_{n}\right\}_{n \geq 1}$ is bounded in $W_{T_{*}}$, in particular, there exists a subsequence $\left\{\bar{w}_{k}\right\}$ of $\left\{\bar{w}_{n}\right\}$ such that:

$$
\begin{aligned}
\bar{w}_{k} \rightarrow \bar{w} & \text { in } L^{2}\left(0, T_{*} ; D(A) \cap V\right), \\
\partial_{t} \bar{w}_{k} \rightarrow \partial_{t} \bar{w} & \text { in } L^{2}\left(0, T_{*} ; H\right) .
\end{aligned}
$$

Then, applying a compactness result of Aubin-Lions type [13]:

$$
\bar{w}_{k} \longrightarrow \bar{w} \quad \text { in } C\left(\left[0, T_{*}\right] ; H\right) .
$$

Therefore, since $\bar{w}_{k}(0)=u_{0}, \forall k \geq 1$, then $\bar{w}(0)=u_{0}$. By lower semi-continuity of the norm,

$$
\begin{aligned}
& \|\bar{w}\|_{L^{\infty}(V)}^{2}+\|\bar{w}\|_{L^{2}(D(A))}^{2}+\left\|\partial_{t} \bar{w}\right\|_{L^{2}(H)}^{2} \\
& \leq \liminf _{k \rightarrow+\infty}\left(\left\|\bar{w}_{k}\right\|_{L^{\infty}(V)}^{2}+\left\|\bar{w}_{k}\right\|_{L^{2}(D(A))}^{2}+\left\|\partial_{t} \bar{w}_{k}\right\|_{L^{2}(H)}^{2}\right) \leq R^{2},
\end{aligned}
$$

then $\bar{w} \in Y\left(T_{*}\right)$, hence $Y\left(T_{*}\right)$ is closed in $X_{T_{*}}$. This one, jointly with 2), imply that $Y\left(T_{*}\right)$ is compact in $X_{T_{*}}$.

4) $\Phi: Y\left(T_{*}\right) \longrightarrow Y\left(T_{*}\right)$ is continuous respect to $X_{T_{*}}$ topology.

Let $\left\{\bar{w}_{n}\right\}_{n \geq 1} \subseteq Y\left(T_{*}\right)$ such that $\bar{w}_{n} \rightarrow \bar{w}$ strongly in $X_{T_{*}}$. Let us prove that:

$$
\Phi\left(\bar{w}_{n}\right)=w_{n} \longrightarrow \Phi(\bar{w})=w \quad \text { strongly in } X_{T_{*}} .
$$

As also $\left\{w_{n}\right\}_{n \geq 1} \subseteq Y\left(T_{*}\right)$, there are subsequences $\left\{\bar{w}_{k}\right\}$ of $\left\{\bar{w}_{n}\right\}$ and $\left\{w_{k}\right\}$ of $\left\{w_{n}\right\}$ such that

$$
\begin{aligned}
& \bar{w}_{k} \rightarrow \bar{w}, \quad \bar{w} \in W_{T_{*}} \\
& w_{k} \rightarrow \tilde{w}, \quad \tilde{w} \in W_{T_{*}}
\end{aligned}
$$

(where the above convergences are as in (42)).

If we consider the system verified by $w_{k}$ and we pass to the limit as $k \rightarrow+\infty$, we obtain that $\tilde{w}$ is a solution of the problem $\left(Q_{l}\right)$ with second member $G(\bar{w}, v)$. By uniqueness $\tilde{w}=\Phi(\bar{w})=w$. Therefore, $w_{k} \longrightarrow w$ weakly in $W_{T_{*}}$ and, by compactness, $w_{k} \longrightarrow w$ in $X_{T_{*}}$. Finally, all the sequence converges.

5) Existence of a fixed point. As $Y\left(T_{*}\right)$ is a convex compact set of $X_{T_{*}}$ and $\Phi$ is continuous respect to $X_{T_{*}}$ topology, applying the Schauder's Theorem we deduce the existence of a fixed point $w$ of $\Phi$ in $Y\left(T_{*}\right)$. Therefore, $w$ is a strong solution of $(Q)$ in $\left(0, T_{*}\right)$ (if $T_{*}$ verifies jointly with $D_{\max }$ the conditions 1 and 3 ). 
Remark 6.1 Again, in the 3D case we cannot bound the nonlinear vertical convection $\left\|\bar{w}_{3} \partial_{z} \bar{w}\right\|_{L^{2}(\Omega)}^{2}$ in function of the strong regularity. Concretely, we obtain a bound of the form

$$
\|\bar{w}\|_{D(A)}^{3}\|\bar{w}\|_{V}
$$

which cannot be bounded using the definition of $Y(T)$. Therefore, we cannot continue with the Fixed Point reasoning.

\section{Uniqueness of weak/strong solution (proof of The- orem 1.6)}

We start from a weak solution $u$ of the system $(E P)$ (see definition 1.1), in particular $u$ verifies the energy inequality:

$$
\begin{aligned}
& \frac{1}{2}\|u(t)\|_{L^{2}(\Omega)}^{2}+\int_{0}^{t}\left(\nu_{h}\left\|\partial_{x} u\right\|_{L^{2}(\Omega)}^{2}+\nu_{v}\left\|\partial_{z} u\right\|_{L^{2}(\Omega)}^{2}\right) d s \\
& \quad \leq \frac{1}{2}\left\|u_{0}\right\|_{L^{2}(\Omega)}^{2}+\int_{0}^{t}\langle F, u\rangle_{\Omega} d s+\int_{0}^{t}\langle\tau, u\rangle_{\Gamma_{s}} d s, \quad \text { a.e. } t \in(0, T) .
\end{aligned}
$$

Suppose that there exists another weak solution $\bar{u}$ more regular (associated to the same data $u_{0}$ and $F$ ). The idea is to find under what regularity conditions, only on $\bar{u}$, we have that $u \equiv \bar{u}$. Observe that, starting from the weak variational formulation of $u$ (definition $1.1)$, it is easy to verify that $\partial_{t} u \in L^{4 / 3}\left(0, T ; W^{\prime}\right)$, where $W=\left\{\psi \in V ; \partial_{z} \psi \in L^{4}(\Omega)\right\}$. In fact, if we want to take $\varphi=\bar{u}$ as test function in the weak variational formulation of $u$, the unique term that presents problems is $\int_{\Omega} u_{3} \partial_{z} \bar{u} u d \Omega$. Then, with the additional regularity of the Theorem 1.6 for $\bar{u}$ (recall $\partial_{z} \bar{u} \in L^{4}\left(0, T ; L^{4}(\Omega)\right)$ ) this term has a sense, hence one verifies the following equality: a.e. $t \in(0, T)$,

$$
\begin{aligned}
& \langle u(t), \bar{u}(t)\rangle_{\Omega}-\int_{0}^{t}\left\langle\partial_{t} \bar{u}, u\right\rangle_{\Omega} d s+\int_{0}^{t} \int_{\Omega}\left(\nu_{h} \partial_{x} u \partial_{x} \bar{u}+\nu_{v} \partial_{z} u \partial_{z} \bar{u}\right) d \Omega d s \\
& =\left\|u_{0}\right\|_{L^{2}(\Omega)}^{2}+\int_{0}^{t}\langle F, \bar{u}\rangle_{\Omega} d s+\int_{0}^{t} \int_{\Omega}\left(u \partial_{x} \bar{u}+u_{3} \partial_{z} \bar{u}\right) u d \Omega d s+\int_{0}^{t}\langle\tau, \bar{u}\rangle_{\Gamma_{s}} d s .
\end{aligned}
$$

Now, we write the differential system for $\left(\bar{u}, \overline{p_{s}}\right)$ as:

$$
\begin{gathered}
\partial_{t} \bar{u}+u \partial_{x} \bar{u}+u_{3} \partial_{z} \bar{u}-\nu_{h} \partial_{x x}^{2} \bar{u}-\nu_{v} \partial_{z z}^{2} \bar{u}+\partial_{x} \overline{p_{s}} \\
=F+(u-\bar{u}) \partial_{x} \bar{u}+\left(u_{3}-\bar{u}_{3}\right) \partial_{z} \bar{u} .
\end{gathered}
$$

Thanks to the additional regularity of $\bar{u}$, we can multiply (45) by $u$ and integrate on $\Omega \times(0, t):$

$$
\begin{aligned}
& \int_{0}^{t}\left\langle\partial_{t} \bar{u}, u\right\rangle_{\Omega} d s+\int_{0}^{t} \int_{\Omega}\left\{\left(u \partial_{x} \bar{u}+u_{3} \partial_{z} \bar{u}\right) u+\nu_{h} \partial_{x} \bar{u} \partial_{x} u+\nu_{v} \partial_{z} \bar{u} \partial_{z} u\right\} d \Omega d s \\
& \quad=\int_{0}^{t}\langle F, u\rangle_{\Omega} d s+\int_{0}^{t} \int_{\Omega}\left((u-\bar{u}) \partial_{x} \bar{u}+\left(u_{3}-\bar{u}_{3}\right) \partial_{z} \bar{u}\right) u d \Omega d s+\int_{0}^{t}\langle\tau, u\rangle_{\Gamma_{s}} d s
\end{aligned}
$$


Adding (44) and (46), the terms $\int_{0}^{t}\left\langle\partial_{t} \bar{u}, u\right\rangle_{\Omega} d s$ and $\int_{0}^{t} \int_{\Omega}\left(u \partial_{x} \bar{u}+u_{3} \partial_{z} \bar{u}\right) u d \Omega d s$ are cancelled, obtaining:

$$
\begin{aligned}
& \langle u(t), \bar{u}(t)\rangle_{\Omega}+\int_{0}^{t} \int_{\Omega} 2\left(\nu_{h} \partial_{x} u \partial_{x} \bar{u}+\nu_{v} \partial_{z} u \partial_{z} \bar{u}\right) d \Omega d s \\
& \quad=\left\|u_{0}\right\|_{L^{2}(\Omega)}^{2}+\int_{0}^{t}\langle F, u+\bar{u}\rangle_{\Omega} d s+\int_{0}^{t}\langle\tau, u+\bar{u}\rangle_{\Gamma_{s}} d s \\
& \quad+\int_{0}^{t} \int_{\Omega}\left((u-\bar{u}) \partial_{x} \bar{u}+\left(u_{3}-\bar{u}_{3}\right) \partial_{z} \bar{u}\right) u d \Omega d s \quad \text { a.e. } t \in(0, T) .
\end{aligned}
$$

Finally, we multiply (45) by $\bar{u}$ and integrate on $\Omega \times(0, t)$, obtaining the energy equality:

$$
\begin{gathered}
\frac{1}{2}\|\bar{u}(t)\|_{L^{2}(\Omega)}^{2}+\int_{0}^{t}\left(\nu_{h}\left\|\partial_{x} \bar{u}\right\|_{L^{2}(\Omega)}^{2}+\nu_{v}\left\|\partial_{z} \bar{u}\right\|_{L^{2}(\Omega)}^{2}\right) d s \\
=\frac{1}{2}\left\|u_{0}\right\|_{L^{2}(\Omega)}^{2}+\int_{0}^{t}\langle F, \bar{u}\rangle_{\Omega} d s+\int_{0}^{t}\langle\tau, \bar{u}\rangle_{\Gamma_{s}} d s \\
\quad+\int_{0}^{t} \int_{\Omega}\left((u-\bar{u}) \partial_{x} \bar{u}+\left(u_{3}-\bar{u}_{3}\right) \partial_{z} \bar{u}\right) \bar{u} d \Omega d s,
\end{gathered}
$$

where the last term on the right hand of (48) vanishes (by the free divergence condition). Then, doing $(43)+(48)-(47)$, we obtain: a.e.t $\in(0, T)$,

$$
\begin{aligned}
& \frac{1}{2}\|u(t)-\bar{u}(t)\|_{L^{2}(\Omega)}^{2}+\int_{0}^{t}\|u(s)-\bar{u}(s)\|_{V}^{2} d s \\
& \quad \leq-\int_{0}^{t} \int_{\Omega}\left((u-\bar{u}) \partial_{x} \bar{u}+\left(u_{3}-\bar{u}_{3}\right) \partial_{z} \bar{u}\right) u d \Omega d s \\
& =-\int_{0}^{t} \int_{\Omega}\left((u-\bar{u}) \partial_{x} \bar{u}+\left(u_{3}-\bar{u}_{3}\right) \partial_{z} \bar{u}\right)(u-\bar{u}) d \Omega d s \\
& =-\int_{0}^{t} \int_{\Omega}|u-\bar{u}|^{2} \partial_{x} \bar{u} d \Omega d s-\int_{0}^{t} \int_{\Omega}\left(u_{3}-\bar{u}_{3}\right) \partial_{z} \bar{u}(u-\bar{u}) d \Omega d s \equiv I_{1}+I_{2}
\end{aligned}
$$

We estimate the $I_{i}$-terms (using lemmas of Section 2):

$$
\begin{aligned}
I_{1} & \leq \int_{0}^{t}\left\|\partial_{x} \bar{u}\right\|_{L^{2}(\Omega)}\|(u-\bar{u})\|_{L^{4}(\Omega)}^{2} d s \\
& \leq \int_{0}^{t}\left\|\partial_{x} \bar{u}\right\|_{L^{2}(\Omega)}\|(u-\bar{u})\|_{L^{2}(\Omega)}\|\nabla(u-\bar{u})\|_{L^{2}(\Omega)} d s \\
& \leq \frac{1}{4} \int_{0}^{t}\|(u-\bar{u})\|_{V^{2}}^{2} d s+C \int_{0}^{t}\|(u-\bar{u})\|_{L^{2}(\Omega)}^{2}\left\|\partial_{x} \bar{u}\right\|_{L^{2}(\Omega)}^{2} d s
\end{aligned}
$$




$$
\begin{aligned}
I_{2} & \leq \int_{0}^{t}\|u-\bar{u}\|_{L^{4}(\Omega)}\left\|\partial_{z} \bar{u}\right\|_{L^{4}(\Omega)}\left\|u_{3}-\bar{u}_{3}\right\|_{L^{2}(\Omega)} d s \\
& \leq D_{\max } \int_{0}^{t}\|\nabla(u-\bar{u})\|_{L^{2}(\Omega)}^{1 / 2}\|u(s)-\bar{u}\|_{L^{2}(\Omega)}^{1 / 2}\left\|\partial_{z} \bar{u}\right\|_{L^{4}(\Omega)}\left\|\partial_{x}\left(u_{3}-\bar{u}_{3}\right)\right\|_{L^{2}(\Omega)} d s \\
& \leq C D_{\max } \int_{0}^{t}\|u-\bar{u}\|_{V}^{3 / 2}\|u-\bar{u}\|_{L^{2}(\Omega)}^{1 / 2}\left\|\partial_{z} \bar{u}\right\|_{L^{4}(\Omega)} d s \\
& \leq \frac{1}{4} \int_{0}^{t}\|(u-\bar{u})\|_{L^{2}(\Omega)}^{2} d s+C D_{\max }^{4} \int_{0}^{t}\left\|\partial_{z} \bar{u}\right\|_{L^{4}(\Omega)}^{4}\|u-\bar{u}\|_{L^{2}(\Omega)}^{2} d s
\end{aligned}
$$

Hence, the inequality (49) becomes: a.e. $t \in(0, T)$,

$$
\begin{aligned}
& \|u(t)-\bar{u}(t)\|_{L^{2}(\Omega)}^{2}+\int_{0}^{t}\|u(s)-\bar{u}(s)\|_{V}^{2} d s \\
& \quad \leq C \int_{0}^{t}\left(\left\|\partial_{x} \bar{u}(s)\right\|_{L^{2}(\Omega)}^{2}+D_{\max }^{4}\left\|\partial_{z} \bar{u}(s)\right\|_{L^{4}(\Omega)}^{4}\right)\|u(s)-\bar{u}(s)\|_{L^{2}(\Omega)}^{2} d s
\end{aligned}
$$

Then, from Gronwall lemma, we can conclude the uniqueness.

Remark 7.1 In the 3D case, the bound obtaining for $I_{2}$ is

$$
\frac{1}{4} \int_{0}^{t}\|\vec{u}(s)-\overrightarrow{\vec{u}}(s)\|_{V}^{2} d s+C \int_{0}^{t}\left\|\partial_{z} \overrightarrow{\vec{u}}(s)\right\|_{L^{4}(\Omega)}^{8}\|\vec{u}(s)-\overrightarrow{\vec{u}}(s)\|_{L^{2}(\Omega)}^{2} d s .
$$

Now, to obtain uniqueness, we have to impose in $\overrightarrow{\vec{u}}$ the following additional regularity

$$
\partial_{z} \overrightarrow{\vec{u}} \in L^{8}\left(0, T ; L^{4}(\Omega)^{2}\right),
$$

which it is not a consequence of the strong regularity.

\section{References}

[1] J. L. Lions, R. Teman, S. Wang, New formulation of the primitive equations of the atmosphere and applications. Nonlinearity, 5, 1992, 237-288.

[2] J. L. Lions, R. Teman, S. Wang, On the equations of the large scale Ocean. Nonlinearity, 5, 1992, 1007-1053.

[3] R. Lewandowski, Analyse Mathématique et Océanographie, Masson, 1997.

[4] J. Pedlosky, Geophysical fluid dynamics, Springer-Verlag, 1987.

[5] O. Besson \& M. R. Laydi, Some Estimates for the Anisotropic Navier-Stokes Equations and for the Hydrostatic Approximation, M²AN-Mod. Math. Ana. Nume., Vol. 7, 1992, 855-865.

[6] P. Azérad \& F. Guillén, Équations de Navier-Stokes en bassin peu profond: l'approximation hydrostatique, C. R. Acad. Sci. Paris, t.329, Série I, 1999, 961-966.

[7] T. Chacón \& F. Guillén, An intrinsic analysis of existence of solutions for the hydrostatic approximation of Navier-Stokes equations, submitted.

[8] F. Guillén \& M. V. Redondo, Convergencia de algunos esquemas numéricos hacia el modelo evolutivo de Ecuaciones Primitivas, Actas XVI CEDYA, VI CMA, University of Las Palmas de Gran Canaria $1999,1165-1172$. 
[9] F. Guillén \& M. V. Redondo, work in preparation.

[10] M. Ziane, Regularity Results for Stokes Type Systems. Applicable Analysis, Vol. 58, 1995, 263-292.

[11] L. Nirenberg, On Elliptic Partial Differential Equations, Ann. Scuo. Norm. Sup. Pisa, 13 (3), 1959, 115-162.

[12] R. A. Adams Sobolev spaces, Academic Press, New York, 1975.

[13] J. L. Lions, Quelques Méthodes de Résolution des Problèmes aux Limites Non Linéaires, Dunod, Paris, 1969. 